\title{
Influence of non-magnetic impurity scattering on the spin dynamics in diluted magnetic semiconductors
}

\author{
M. Cygorek,${ }^{1}$ F. Ungar, ${ }^{1}$ P. I. Tamborenea, ${ }^{2}$ and V. M. Axt ${ }^{1}$ \\ ${ }^{1}$ Theoretische Physik III, Universität Bayreuth, 95440 Bayreuth, Germany \\ ${ }^{2}$ Departamento de Física and IFIBA, FCEN, Universidad de Buenos Aires, \\ Ciudad Universitaria, Pabellón I, 1428 Ciudad de Buenos Aires, Argentina
}

\begin{abstract}
The doping of semiconductors with magnetic impurities gives rise not only to a spin-spin interaction between quasi-free carriers and magnetic impurities, but also to a local spin-independent disorder potential for the carriers. Based on a quantum kinetic theory for the carrier and impurity density matrices as well as the magnetic and non-magnetic carrier-impurity correlations, the influence of the non-magnetic scattering potential on the spin dynamics in DMS after optical excitation with circularly polarized light is investigated using the example of Mn-doped CdTe. It is shown that non-Markovian effects, which are predicted in calculations where only the magnetic carrier-impurity interaction is accounted for, can be strongly suppressed in the presence of non-magnetic impurity scattering. This effect can be traced back to a significant redistribution of carriers in $\mathbf{k}$-space which is enabled by the build-up of large carrier-impurity correlation energies. A comparison with the Markov limit of the quantum kinetic theory shows that, in the presence of an external magnetic field parallel to the initial carrier polarization, the asymptotic value of the spin polarization at long times is significantly different in the quantum kinetic and the Markovian calculations. This effect can also be attributed to the formation of strong correlations which invalidates the semiclassical Markovian picture and it is stronger when the non-magnetic carrier-impurity interaction is accounted for. In an external magnetic field perpendicular to the initial carrier spin, the correlations are also responsible for a renormalization of the carrier spin precession frequency. Considering only the magnetic carrier-impurity interaction, a significant renormalization is predicted for a very limited set of material parameters and excitation conditions. Accounting also for the non-magnetic interaction a relevant renormalization of the precession frequency is found to be more ubiquitous.
\end{abstract}

PACS numbers: 75.78.Jp, 75.50.Pp, 75.30.Hx, 72.10.Fk

\section{INTRODUCTION}

Most of the devices based on the spintronics paradigm that are commercially available today use the fact that spin-up and spin-down carriers exhibit different transmission and reflection probabilities at interfaces involving ferromagnetic metals ${ }^{1,2}$. However, some applications, like spin transistors ${ }^{3}$, require the control not only of spinup and spin-down occupations, but also of the coherent precession of spins perpendicular to the quantization axis provided by the structure. For this purpose, spintronic devices based on semiconductors are preferable to metallic structures since the dephasing time in a metal is about three orders of magnitude shorter than in a semiconductor ${ }^{4}$. In the context of semiconductor spintronics ${ }^{5-7}$, a particularly interesting class of materials for future applications are diluted magnetic semiconductors (DMS) ${ }^{8-22}$, which are obtained when semiconductors are doped with transition metal elements, such as Mn, which act as localized magnetic moments. While some types of DMS, such as $\mathrm{Ga}_{1-x} \mathrm{Mn}_{x} \mathrm{As}$, exhibit a ferromagnetic phase ${ }^{8,23}$, other types of DMS, like the usually paramagnetic CdMnTe, are especially valued for the enhancement of the effective carrier g-factor by the giant Zeeman effect that can be used, e.g., to facilitate an injection of a spin-polarized current into a light-emitting diode ${ }^{24}$. Besides causing the giant Zeeman effect, the $s-d$ exchange interaction between the quasi-free carriers and localized magnetic impurities also leads to other effects, such as inducing spin-flip scattering and thereby a direct transfer of spins from the carriers to the impurities and vice versa ${ }^{25-28}$.

Typically, the $s$ - $d$ interaction is described by a Kondolike $^{29}$ localized spin-spin interaction between carriers and impurities. However, in real DMS materials, the introduction of $\mathrm{Mn}$ impurities not only leads to a spindependent interaction Hamiltonian, but also to a spinindependent local potential for the carriers ${ }^{30}$. The reason for the appearance of this spin-independent potiential is that, in the case of $\mathrm{Cd}_{1-x} \mathrm{Mn}_{x} \mathrm{Te}$, the semiconductor CdTe has a different band structure than MnTe and carriers located at unit cells with $\mathrm{Mn}$ impurities experience a larger local potiential energy than carriers at unit cells with $\mathrm{Cd}$ cations. The strength of this local potential can be estimated by the conduction and valence band offsets between CdTe and MnTe. Note, however, that usually, CdTe crystallizes in a zinc-blende structure, while MnTe is found in a wurzite structure. Thus, a better estimation for the strength of the spin-independent local potential is obtained by studying $\mathrm{CdTe} / \mathrm{Cd}_{1-x} \mathrm{Mn}_{x}$ Te heterostructures where both materials appear in the form of a zinc-blende lattice ${ }^{31}$. From such investigations, the strenght of the local spin-independent potential for carriers at $\mathrm{Mn}$ sites of about $1.6 \mathrm{eV}$ can be estimated. In contrast, the spin-dependent local interaction in DMS is typically about $220 \mathrm{meV}$, i.e. one order of magnitude lower. This consideration suggests that the non-magnetic 
impurity scattering caused by the local spin-independent interaction between carriers and impurities should not be disregarded in the study of the spin physics in DMS.

It is noteworthy that a theory which takes into account a local magnetic interaction as well as a nonmagnetic local potential in a DMS, the V-J tight-binding model was employed to study the magnetic properties of $\mathrm{GaMnAs}^{32}$ and it was found that taking into account the non-magnetic interaction is necessary in order to obtain results in good quantitative agreement with ab initio calculations for the Curie temperature and with experimental data for the optical conductivity.

For the spin dynamics, scattering at non-magnetic impurities has already important consequences in nonmagnetic semiconductors ${ }^{33}$ in the presence of spin-orbit fields, where scattering processes can enhance or reduce the spin relaxation and dephasing significantly, e.g., via the Elliott-Yafet ${ }^{34}$ and D'yakonov-Perel ${ }^{35}$ mechanisms.

The goal of the present article is to investigate how the non-magnetic interaction between carriers and impurities affects the spin dynamics in paramagnetic II-VI DMS. To this end we employ a quantum kinetic theory for carrier and impurity density matrices including the carrierimpurity correlations starting from a system Hamiltonian that comprises a kinetic energy term, the magnetic and non-magnetic carrier-impurity interactions as well as the carrier and impurity Zeeman energies. Earlier quantum kinetic studies of the spin dynamics in $\mathrm{DMS}^{25-27,36,37}$, which only considered the spin-dependent $s$ - $d$ interaction, predicted that in some cases, such as in narrow quantum wells optically excited very close to the band edge $^{38}$, the spin transfer between carriers and impurities cannot be well described by rate equations. Rather, the time evolution of the carrier spin is, in these cases, nonexponential and it can exhibit non-monotonic features such as overshoots. These effects are non-Markovian, as they can be traced back to the finite memory provided by the correlations, since the Markovian assumption of a $\delta$ like memory leads to effective rate equations that predict an exponential spin dynamics ${ }^{28}$.

Here, we find that these non-Markovian effects predicted in the theory of Refs. 25-27, 36, and 37 are suppressed in the case of the conduction band of a $\mathrm{Cd}_{1-x} \mathrm{Mn}_{x}$ Te quantum well when non-magnetic scattering of carriers at the impurities is taken into account. While, in this case, the non-monotonic behavior of the spin dynamics disappears, the quantum kinetic theory predicts quantitative changes in the effective spin transfer rate compared with the Fermi's golden rule value. The suppression of the non-Markovian features is mainly caused by a significant redistribution of carriers away from the band edge where the non-Markovian effects are particularly strong ${ }^{38}$. This carrier redistribution is facilitated by the build-up of strong carrier-impurity correlations providing a correlation energy of the order of a few $\mathrm{meV}$ per electron that leads to an increase of the average kinetic electron energy by about the same amount. Due to the different strengths of the interactions in the con- duction band of $\mathrm{Cd}_{1-x} \mathrm{Mn}_{x} \mathrm{Te}$, the non-magnetic carrierimpurity correlation energy is also much larger than the magnetic correlation energy studied before in Ref. 39 .

In other cases, such as in the valence band of $\mathrm{Cd}_{1-x} \mathrm{Mn}_{x} \mathrm{Te}$, the non-magnetic impurty scattering can be much weaker than the magnetic spin-flip scattering and the non-Markovian effects prevail.

In the presence of an external magnetic field parallel to the initial carrier spin polarization, it was shown ${ }^{40}$ that a quantum kinetic treatment of the magnetic part of the carrier-impurity interaction in DMS leads to a significantly different asymptotic value of the carrier spin polarization at long times $t$. Because this is also a consequence of an energetic redistribution of carriers, including non-magnetic scattering increases this effect. If the initial carrier spin polarization is perpendicular to the external magnetic field, the carrier spins precess about the effective field comprised of the external field and the mean field due to the impurity magnetization. As shown in Ref. 39, the carrier-impurity correlations built up by the magnetic $s$ - $d$ interaction renormalize the carrier spin precession frequency. Here, we show that when both, the magnetic and the non-magnetic interactions are taken into account the renormalization of the carrier spin precession frequency can be different in sign and magnitude compared with calculations in which only the magnetic interaction is considered.

The article is structured as follows: First, quantum kinetic equations of motion for the carrier and impurity density matrices as well as for the magnetic and nonmagnetic carrier-impurity correlations are formulated for a DMS with magnetic and non-magnetic carrier-impurity interactions. Then, we derive the Markov limit of the quantum kinetic theory which enables a comparison and allows us to distinguish the genuine quantum kinetic effects from the Markovian behavior. Furthermore, from the Markov limit we can derive analytic expressions for the carrier-impurity correlation energies as well as the correlation-induced renormalization of the carrier spin precession frequency. After having layed out the theory, we present numerical simulations of the quantum kinetic equations for the conduction band of a $\mathrm{Cd}_{1-x} \mathrm{Mn}_{x} \mathrm{Te}$ quantum well including magnetic and non-magnetic scattering at the Mn impurities and discuss the energetic redistribution of carriers as well as the correlation energies. Then, we estimate the influence of non-magnetic impurity interaction on the spin dynamics in the valence band of $\mathrm{Cd}_{1-x} \mathrm{Mn}_{x}$ Te. Finally, we discuss the effects of the non-magnetic impurity scattering on the spin dynamics in DMS in the presence of an external magnetic field parallel and perpendicular to an initial non-equilibrium carrier spin polarization. 


\section{THEORY}

\section{A. DMS Hamiltonian}

Here, we consider an intrinsic DMS such as $\mathrm{Cd}_{1-x} \mathrm{Mn}_{x} \mathrm{Te}$ in the presence of an external magnetic field. The total Hamiltonian of this DMS is given by

$$
\begin{aligned}
H & =H_{0}+H_{\mathrm{sd}}+H_{\mathrm{imp}}+H_{Z}^{\mathrm{e}}+H_{Z}^{\mathrm{Mn}}, \\
H_{0} & =\sum_{\mathbf{k} \sigma} \hbar \omega_{\mathbf{k}} c_{\sigma \mathbf{k}}^{\dagger} c_{\sigma \mathbf{k}}, \\
H_{\mathrm{sd}} & =\frac{J_{\mathrm{sd}}}{V} \sum_{\mathbf{k} \mathbf{k}^{\prime} \sigma \sigma^{\prime}} \sum_{I n n^{\prime}} \mathbf{S}_{n n^{\prime}} \cdot \mathbf{s}_{\sigma \sigma^{\prime}} c_{\sigma \mathbf{k}}^{\dagger} c_{\sigma^{\prime} \mathbf{k}^{\prime}} e^{i\left(\mathbf{k}^{\prime}-\mathbf{k}\right) \mathbf{R}_{I}} \hat{P}_{n n^{\prime}}^{I}, \\
H_{\mathrm{imp}} & =\frac{J_{0}}{V} \sum_{\mathbf{k} \mathbf{k}^{\prime} \sigma} \sum_{J} c_{\sigma \mathbf{k}}^{\dagger} c_{\sigma \mathbf{k}^{\prime}} e^{i\left(\mathbf{k}^{\prime}-\mathbf{k}\right) \mathbf{R}_{J}}, \\
H_{Z}^{\mathrm{e}} & =\sum_{\mathbf{k} \sigma \sigma^{\prime}} \hbar g_{e} \mu_{B} \mathbf{B} \cdot \mathbf{s}_{\sigma \sigma^{\prime}} c_{\sigma \mathbf{k}}^{\dagger} c_{\sigma^{\prime} \mathbf{k}}, \\
H_{Z}^{\mathrm{Mn}} & =\sum_{I n n^{\prime}} \hbar g_{\mathrm{Mn}} \mu_{B} \mathbf{B} \cdot \mathbf{S}_{n n^{\prime}} \hat{P}_{n n^{\prime}}^{I},
\end{aligned}
$$

where $H_{0}$ is the single-electron Hamiltonian due to the crystal potential, $H_{\text {sd }}$ describes the magnetic $s$ - $d$ exchange interaction between the carriers and the impurities, $H_{\mathrm{imp}}$ describes the spin-independent scattering of carriers at impurities and $H_{Z}^{\mathrm{e}}$ and $H_{Z}^{\mathrm{Mn}}$ are the carrier and impurity Zeeman energies.

In Eqs. (1), $c_{\sigma \mathbf{k}}^{\dagger}$ and $c_{\sigma \mathbf{k}}$ denote the creation and annihilation operators for conduction band electrons with wave vector $\mathbf{k}$ in the spin subband $\sigma=\{\uparrow, \downarrow\}$. The magnetic Mn impurities are described by the operator $\hat{P}_{n n^{\prime}}^{I}=\lfloor I, n\rangle\left\langle I, n^{\prime}\right\rfloor$ where $|I, n\rangle$ is the $n$-th spin state $\left(n \in\left\{-\frac{5}{2},-\frac{3}{2}, \ldots \frac{5}{2}\right\}\right)$ of the $I$-th magnetic impurity located at $\mathbf{R}_{I}$. The band structure of the semiconductor is described by $\hbar \omega_{\mathbf{k}}$, which we assume to be parabolic $\omega_{\mathbf{k}}=\frac{\hbar \mathbf{k}^{2}}{2 m^{*}}$ with effective mass $m^{*} . \quad V$ denotes the volume of the sample. $J_{\text {sd }}$ is the $s$ - $d$ coupling constant for the spin-spin interaction between carriers and impurities and $J_{0}$ is the non-magnetic coupling constant. $\mathbf{S}_{n_{1} n_{2}}$ and $\mathbf{S}_{\sigma_{1} \sigma_{2}}$ are the vectors with components consisting of spin$\frac{5}{2}$ and spin- $\frac{1}{2}$ spin matrices for the impurities and the conduction band electrons, respectively, where the unit $\hbar$ has been substituted into the definition of $J_{\text {sd }}$ so that $\mathbf{s}_{\sigma_{1} \sigma_{2}}=\frac{1}{2} \boldsymbol{\sigma}_{\sigma_{1} \sigma_{2}}$, where $\boldsymbol{\sigma}_{\sigma_{1} \sigma_{2}}$ are the Pauli matrices. Finally, $g_{e}$ and $g_{\mathrm{Mn}}$ are the g-factors of the electrons and the impurities, respectively, and $\mu_{B}$ is the Bohr magneton.

In order to account for spin-independent scattering not only at $\mathrm{Mn}$ impurities but also additional nonmagnetic scattering centers, such as in quaternary compound DMSs like $\mathrm{HgCdMnTe}{ }^{41}$, we allow the number of scattering centers $N_{\text {imp }}$ in general to be larger than the number of magnetic impurities $N_{\mathrm{Mn}}$. Here, we use the notation that the index $I$ runs from 1 to $N_{\mathrm{Mn}}$ while the index $J$ runs from 1 to $N_{\text {imp }}$.

\section{B. Quantum kinetic equations of motion}

The goal of this artilce is to study the time evolution of the carrier spin polarization after optical excitation with circularly polarized light which can be extracted from the carrier density matrix. In this section, we derive the corresponding equations of motion starting from the total Hamiltonian in Eqs. (1).

Following Ref. 36, where for the conduction band only $H_{0}$ and $H_{\text {sd }}$ in Eqs. (1) were considered, we seek to obtain a closed set of equations for the reduced carrier and impurity density matrices as well as for the carrier-impurity correlations:

$$
\begin{aligned}
M_{n_{1}}^{n_{2}} & =\left\langle\hat{P}_{n_{1} n_{2}}^{I}\right\rangle & \\
C_{\sigma_{1} \mathbf{k}_{1}}^{\sigma_{2}} & =\left\langle c_{\sigma_{1} \mathbf{k}_{1}}^{\dagger} c_{\sigma_{2} \mathbf{k}_{1}}\right\rangle, & (2 \mathrm{a}) \\
\bar{C}_{\sigma_{1} \mathbf{k}_{1}}^{\sigma_{2} \mathbf{k}_{2}} & =V\left\langle c_{\sigma_{1} \mathbf{k}_{1}}^{\dagger} c_{\sigma_{2} \mathbf{k}_{2}} e^{i\left(\mathbf{k}_{2}-\mathbf{k}_{1}\right) \mathbf{R}_{J}}\right\rangle, & \text { for } \mathbf{k}_{2} \neq \mathbf{k}_{1}, \\
Q_{\sigma_{1} n_{1} \mathbf{k}_{1}}^{\sigma_{2} n_{2} \mathbf{k}_{2}} & =V\left\langle c_{\sigma_{1} \mathbf{k}_{1}}^{\dagger} c_{\sigma_{2} \mathbf{k}_{2}} e^{i\left(\mathbf{k}_{2}-\mathbf{k}_{1}\right) \mathbf{R}_{I}} \hat{P}_{n_{1} n_{2}}^{I}\right\rangle, & \text { for } \mathbf{k}_{2} \neq \mathbf{k}_{1} .
\end{aligned}
$$

$M_{n_{1}}^{n_{2}}$ and $C_{\sigma_{1} \mathbf{k}_{1}}^{\sigma_{2}}$ are the impurity and electron density matrices and $\bar{C}_{\sigma_{1} \mathbf{k}_{1}}^{\sigma_{2} \mathbf{k}_{2}}$ as well as $Q_{\sigma_{1} n_{1} \mathbf{k}_{1}}^{\sigma_{2} n_{2} \mathbf{k}_{2}}$ are the non-magnetic and magnetic carrier-impurity correlations, respectively. In Eqs. (2), the brackets denote not only the quantum mechanical average of the operators, but also an average over a random distribution of impurity positions, which we assume to be on average homogeneous so that $\left\langle e^{i\left(\mathbf{k}_{2}-\mathbf{k}_{1}\right) \mathbf{R}_{J}}\right\rangle=\delta_{\mathbf{k}_{1} \mathbf{k}_{2}}$.

The equations of motion for the variables defined in Eqs. (2) can be derived using the Heisenberg equations of motion for the corresponding operators. Note, however, that this procedure leads to an infinite hierarchy of variables and equations of motion, since, e. g., the equation of motion for $\left\langle c_{\sigma_{1} \mathbf{k}_{1}}^{\dagger} c_{\sigma_{2} \mathbf{k}_{2}} e^{i\left(\mathbf{k}_{2}-\mathbf{k}_{1}\right) \mathbf{R}_{I}} \hat{P}_{n_{1} n_{2}}^{I}\right\rangle$ contains also terms of the form $\left\langle c_{\sigma_{1} \mathbf{k}_{1}}^{\dagger} c_{\sigma \mathbf{k}} e^{i\left(\mathbf{k}-\mathbf{k}_{1}\right) \mathbf{R}_{I}} e^{i\left(\mathbf{k}_{2}-\mathbf{k}\right) \mathbf{R}_{I^{\prime}}} \hat{P}_{n_{1} n_{2}}^{I} \hat{P}_{n n^{\prime}}^{I^{\prime}}\right\rangle$ for $I^{\prime} \neq I$ which cannot be expressed in terms of the variables in Eqs. (2). Thus, in order to obtain a closed set of equations, one has to employ a truncation scheme. Here, we follow the procedure of Ref. 36: we factorize the averages over products of operators and define the true correlations to be the remainder when all combinations of factorizations have been subtracted from the averages. For example, we define (for $\mathbf{k}_{2} \neq \mathbf{k}_{1}$ )

$$
\begin{aligned}
& \delta\left\langle c_{\sigma_{1} \mathbf{k}_{1}}^{\dagger} c_{\sigma_{2} \mathbf{k}_{2}} e^{i\left(\mathbf{k}_{2}-\mathbf{k}_{1}\right) \mathbf{R}_{I}} \hat{P}_{n_{1} n_{2}}^{I}\right\rangle:= \\
& \left\langle c_{\sigma_{1} \mathbf{k}_{1}}^{\dagger} c_{\sigma_{2} \mathbf{k}_{2}} e^{i\left(\mathbf{k}_{2}-\mathbf{k}_{1}\right) \mathbf{R}_{I}} \hat{P}_{n_{1} n_{2}}^{I}\right\rangle \\
& -\left(\left\langle c_{\sigma_{1} \mathbf{k}_{1}}^{\dagger} c_{\sigma_{2} \mathbf{k}_{2}}\right\rangle\left\langle e^{i\left(\mathbf{k}_{2}-\mathbf{k}_{1}\right) \mathbf{R}_{I}}\right\rangle\left\langle\hat{P}_{n_{1} n_{2}}^{I}\right\rangle\right. \\
& +\left\langle c_{\sigma_{1} \mathbf{k}_{1}}^{\dagger} c_{\sigma_{2} \mathbf{k}_{2}} e^{i\left(\mathbf{k}_{2}-\mathbf{k}_{1}\right) \mathbf{R}_{I}}\right\rangle\left\langle\hat{P}_{n_{1} n_{2}}^{I}\right\rangle \\
& \left.+\left\langle e^{i\left(\mathbf{k}_{2}-\mathbf{k}_{1}\right) \mathbf{R}_{I}}\right\rangle\left\langle c_{\sigma_{1} \mathbf{k}_{1}}^{\dagger} c_{\sigma_{2} \mathbf{k}_{2}} \hat{P}_{n_{1} n_{2}}^{I}\right\rangle\right)
\end{aligned}
$$

where $\delta\langle\ldots\rangle$ denotes the true correlations. The basic assumption of the truncation scheme of Ref. 36 is that 
all correlations higher than $\delta\left\langle c_{\sigma_{1} \mathbf{k}_{1}}^{\dagger} c_{\sigma_{2} \mathbf{k}_{2}} e^{i\left(\mathbf{k}_{2}-\mathbf{k}_{1}\right) \mathbf{R}_{I}}\right\rangle$ and $\delta\left\langle c_{\sigma_{1} \mathbf{k}_{1}}^{\dagger} c_{\sigma_{2} \mathbf{k}_{2}} e^{i\left(\mathbf{k}_{2}-\mathbf{k}_{1}\right) \mathbf{R}_{I}} \hat{P}_{n_{1} n_{2}}^{I}\right\rangle$ are negligible. This assumption results in a closed set of equations of motion for the reduced density matrices and the true correlations. However, it turns out ${ }^{26}$ that the equations of motion can be written down in a more condensed form when switching back to the full (non-factorized) higher order density matrices as variables, after the higher (true) correlations are neglected. For details of this procedure, the reader is referred to Refs. 26 and 36.

Applying this truncation scheme to the total Hamiltonian (1) including magnetic and non-magnetic carrierimpurity interactions as well as the Zeeman terms for carriers and impurities leads to the equations of motion for the variables defined in Eqs. (2):

$$
\begin{aligned}
-i \hbar \frac{\partial}{\partial t} M_{n_{1}}^{n_{2}}= & \left.\sum_{n} \hbar \boldsymbol{\omega}_{\mathrm{Mn}} \cdot\left(\mathbf{S}_{n n_{1}} M_{n}^{n_{2}}-\mathbf{S}_{n_{2} n} M_{n_{1}}^{n}\right)+\frac{J_{\mathrm{sd}}}{V^{2}} \sum_{n} \sum_{\mathbf{k} \mathbf{k}^{\prime} \sigma \sigma^{\prime}}\left(\mathbf{S}_{n n_{1}} \cdot \mathbf{s}_{\sigma \sigma^{\prime}} Q_{\sigma n \mathbf{k}}^{\sigma^{\prime} n_{2} \mathbf{k}^{\prime}}-\mathbf{S}_{n_{2} n} \cdot \mathbf{s}_{\sigma \sigma^{\prime}} Q_{\sigma n_{1} \mathbf{k}}^{\sigma^{\prime} n \mathbf{k}^{\prime}}\right)\right] \\
-i \hbar \frac{\partial}{\partial t} C_{\sigma_{1} \mathbf{k}_{1}}^{\sigma_{2}}= & \sum_{\sigma} \hbar \boldsymbol{\omega}_{e} \cdot\left(\mathbf{s}_{\sigma \sigma_{1}} C_{\sigma \mathbf{k}_{1}}^{\sigma_{2}}-\mathbf{s}_{\sigma_{2} \sigma} C_{\sigma_{1} \mathbf{k}_{1}}^{\sigma}\right)+J_{\mathrm{sd}} \frac{N_{\mathrm{Mn}}}{V^{2}} \sum_{n n^{\prime}} \sum_{\mathbf{k} \sigma}\left(\mathbf{S}_{n n^{\prime}} \cdot \mathbf{s}_{\sigma \sigma_{1}} Q_{\sigma n \mathbf{k}}^{\sigma_{2} n^{\prime} \mathbf{k}_{1}}-\mathbf{S}_{n n^{\prime}} \cdot \mathbf{s}_{\sigma_{2} \sigma} Q_{\sigma_{1} n \mathbf{k}_{1}}^{\sigma n^{\prime} \mathbf{k}}\right)+ \\
& +J_{0} \frac{N_{\mathrm{imp}}}{V^{2}} \sum_{\mathbf{k}}\left(\bar{C}_{\sigma_{1} \mathbf{k}}^{\sigma_{2} \mathbf{k}_{1}}-\bar{C}_{\sigma_{1} \mathbf{k}_{1}}^{\sigma_{2} \mathbf{k}}\right), \\
-i \hbar \frac{\partial}{\partial t} Q_{\sigma_{1} n_{1} \mathbf{k}_{1}}^{\sigma_{2} n_{2} \mathbf{k}_{2}}= & \hbar\left(\omega_{\mathbf{k}_{1}}-\omega_{\mathbf{k}_{2}}\right) Q_{\sigma_{1} n_{1} \mathbf{k}_{1}}^{\sigma_{2} n_{2} \mathbf{k}_{2}}+b_{\sigma_{1} n_{1} \mathbf{k}_{1}}^{\sigma_{2} n_{2} \mathbf{k}_{2} I}+b_{\sigma_{1} n_{1} \mathbf{k}_{1}}^{\sigma_{2} n_{2} \mathbf{k}_{2} I I}+b_{\sigma_{1} n_{1} \mathbf{k}_{1}}^{\sigma_{2} n_{2} \mathbf{k}_{2} I I I}+b_{\sigma_{1} n_{1} \mathbf{k}_{1}}^{\sigma_{2} n_{2} \mathbf{k}_{2} \operatorname{imp}} \\
-i \hbar \frac{\partial}{\partial t} \bar{C}_{\sigma_{1} \mathbf{k}_{1}}^{\sigma_{2} \mathbf{k}_{2}}= & \hbar\left(\omega_{\mathbf{k}_{1}}-\omega_{\mathbf{k}_{2}}\right) \bar{C}_{\sigma_{1} \mathbf{k}_{1}}^{\sigma_{2} \mathbf{k}_{2}}+c_{\sigma_{1} \mathbf{k}_{1}}^{\sigma_{2} \mathbf{k}_{2} I}+c_{\sigma_{1} \mathbf{k}_{1}}^{\sigma_{2} \mathbf{k}_{2} I I}+c_{\sigma_{1} \mathbf{k}_{1}}^{\sigma_{2} \mathbf{k}_{2} I I}+c_{\sigma_{1} \mathbf{k}_{1}}^{\sigma_{2} \mathbf{k}_{2}} \mathrm{sd}^{I I I}
\end{aligned}
$$

with

$$
\begin{aligned}
b_{\sigma_{1} n_{1} \mathbf{k}_{1}}^{\sigma_{2} n_{2} \mathbf{k}_{2} I}= & \sum_{n \sigma \sigma^{\prime}} J_{\mathrm{sd}}\left[\mathbf{S}_{n n_{1}} \cdot \mathbf{s}_{\sigma \sigma^{\prime}}\left(\delta_{\sigma_{1} \sigma^{\prime}}-C_{\sigma_{1} \mathbf{k}_{1}}^{\sigma^{\prime}}\right) C_{\sigma \mathbf{k}_{2}}^{\sigma_{2}} M_{n}^{n_{2}}-\mathbf{S}_{n_{2} n} \cdot \mathbf{s}_{\sigma \sigma^{\prime}}\left(\delta_{\sigma \sigma_{2}}-C_{\sigma \mathbf{k}_{2}}^{\sigma_{2}}\right) C_{\sigma_{1} \mathbf{k}_{1}}^{\sigma^{\prime}} M_{n_{1}}^{n}\right] \\
b_{\sigma_{1} n_{1} \mathbf{k}_{1}}^{\sigma_{2} n_{2} \mathbf{k}_{2} I I}= & \sum_{\sigma} \hbar \boldsymbol{\omega}_{e} \cdot\left(\mathbf{s}_{\sigma \sigma_{1}} Q_{\sigma n_{1} \mathbf{k}_{1}}^{\sigma_{2} n_{2} \mathbf{k}_{2}}-\mathbf{s}_{\sigma_{2} \sigma} Q_{\sigma_{1} n_{1} \mathbf{k}_{1}}^{\sigma n_{2} \mathbf{k}_{2}}\right)+\sum_{n} \hbar \boldsymbol{\omega}_{\mathrm{Mn}} \cdot\left(\mathbf{S}_{n n_{1}} Q_{\sigma_{1} n \mathbf{k}_{1}}^{\sigma_{2} n_{2} \mathbf{k}_{2}}-\mathbf{S}_{n_{2} n} Q_{\sigma_{1} n_{1} \mathbf{k}_{1}}^{\sigma_{2} n \mathbf{k}_{2}}\right) \\
b_{\sigma_{1} n_{1} \mathbf{k}_{1}}^{\sigma_{2} n_{2} \mathbf{k}_{2} I I I}= & \frac{J_{\mathrm{sd}}}{V} \sum_{n} \sum_{\mathbf{k} \sigma}\left\{\left(\mathbf{S}_{n n_{1}} \cdot \mathbf{s}_{\sigma \sigma_{1}} Q_{\sigma n \mathbf{k}}^{\sigma_{2} n_{2} \mathbf{k}_{2}}-\mathbf{S}_{n_{2} n} \cdot \mathbf{s}_{\sigma_{2} \sigma} Q_{\sigma_{1} n_{1} \mathbf{k}_{1}}^{\sigma n \mathbf{k}}\right)\right. \\
& \left.-\sum_{\sigma^{\prime}} \mathbf{s}_{\sigma \sigma^{\prime}} \cdot\left[C_{\sigma_{1} \mathbf{k}_{1}}^{\sigma^{\prime}}\left(\mathbf{S}_{n n_{1}} Q_{\sigma n \mathbf{k}}^{\sigma_{2} n_{2} \mathbf{k}_{2}}-\mathbf{S}_{n_{2} n} Q_{\sigma n_{1} \mathbf{k}}^{\sigma_{2} n \mathbf{k}_{2}}\right)+C_{\sigma \mathbf{k}_{2}}^{\sigma_{2}}\left(\mathbf{S}_{n n_{1}} Q_{\sigma_{1} n \mathbf{k}_{1}}^{\sigma^{\prime} n_{2} \mathbf{k}}-\mathbf{S}_{n_{2} n} Q_{\sigma_{1} n_{1} \mathbf{k}_{1}}^{\sigma^{\prime} n \mathbf{k}}\right)\right]\right\} \\
b_{\sigma_{1} n_{1} \mathbf{k}_{1}}^{\sigma_{2} n_{2} \mathbf{k}_{2}} \mathrm{imp}= & J_{0}\left[\left(C_{\sigma_{1} \mathbf{k}_{2}}^{\sigma_{2}}-C_{\sigma_{1} \mathbf{k}_{1}}^{\sigma_{2}}\right) M_{n_{1}}^{n_{2}}+\frac{1}{V} \sum_{\mathbf{k}}\left(Q_{\sigma_{1} n_{1} \mathbf{k}}^{\sigma_{2} n_{2} \mathbf{k}_{2}}-Q_{\sigma_{1} n_{1} \mathbf{k}_{1}}^{\sigma_{2} n_{2} \mathbf{k}}\right)\right]
\end{aligned}
$$

and

$$
\begin{aligned}
c_{\sigma_{1} \mathbf{k}_{1}}^{\sigma_{2} \mathbf{k}_{2} I} & =J_{0}\left(C_{\sigma_{1} \mathbf{k}_{2}}^{\sigma_{2}}-C_{\sigma_{1} \mathbf{k}_{1}}^{\sigma_{2}}\right), \\
c_{\sigma_{1} \mathbf{k}_{1}}^{\sigma_{2} \mathbf{k}_{2} I I} & =\sum_{\sigma} \hbar \boldsymbol{\omega}_{e} \cdot\left(\mathbf{s}_{\sigma \sigma_{1}} \bar{C}_{\sigma \mathbf{k}_{1}}^{\sigma_{2} \mathbf{k}_{2}}-\mathbf{s}_{\sigma_{2} \sigma} \bar{C}_{\sigma_{1} \mathbf{k}_{1}}^{\sigma \mathbf{k}_{2}}\right) \\
c_{\sigma_{1} \mathbf{k}_{1}}^{\sigma_{2} \mathbf{k}_{2} I I I} & =\frac{J_{0}}{V} \sum_{\mathbf{k}}\left(\bar{C}_{\sigma_{1} \mathbf{k}}^{\sigma_{2} \mathbf{k}_{2}}-\bar{C}_{\sigma_{1} \mathbf{k}_{1}}^{\sigma_{2} \mathbf{k}}\right) \\
c_{\sigma_{1} \mathbf{k}_{1}}^{\sigma_{2} \mathbf{k}_{2} \mathrm{sd}} & =J_{\mathrm{sd}} \sum_{n n^{\prime}} \sum_{\sigma} M_{n n^{\prime}} \mathbf{S}_{n n^{\prime}} \cdot\left(\mathbf{s}_{\sigma \sigma_{1}} C_{\sigma \mathbf{k}_{2}}^{\sigma_{2}}-\mathbf{s}_{\sigma_{2} \sigma} C_{\sigma_{1} \mathbf{k}_{1}}^{\sigma}\right)+\frac{J_{\mathrm{sd}}}{V} \frac{N_{\mathrm{Mn}}}{N_{\mathrm{imp}}} \sum_{n n^{\prime}} \sum_{\mathbf{k} \sigma} \mathbf{S}_{n n^{\prime}} \cdot\left(\mathbf{s}_{\sigma \sigma_{1}} Q_{\sigma n \mathbf{k}}^{\sigma_{2} n^{\prime} \mathbf{k}_{2}}-\mathbf{s}_{\sigma_{2} \sigma} Q_{\sigma_{1} n \mathbf{k}_{1}}^{\sigma n^{\prime} \mathbf{k}}\right)
\end{aligned}
$$

where $b_{\sigma_{1} n_{1} \mathbf{k}_{1}}^{\sigma_{2} n_{2} \mathbf{k}_{2} X}$ are the source terms for the magnetic carrier-impurity correlations, $c_{\sigma_{1} \mathbf{k}_{1}}^{\sigma_{2} \mathbf{k}_{2} X}$ are the sources for the non-magnetic correlations and

$$
\begin{aligned}
& \boldsymbol{\omega}_{\mathrm{Mn}}=g_{\mathrm{Mn}} \mu_{B} \mathbf{B}+\frac{J_{\mathrm{sd}}}{\hbar} \frac{1}{V} \sum_{\mathbf{k} \sigma \sigma^{\prime}} \mathbf{s}_{\sigma \sigma^{\prime}} C_{\sigma \mathbf{k}}^{\sigma^{\prime}}, \\
& \boldsymbol{\omega}_{\mathrm{e}}=g_{\mathrm{e}} \mu_{B} \mathbf{B}+\frac{J_{\mathrm{sd}}}{\hbar} \frac{N_{\mathrm{Mn}}}{V} \sum_{n n^{\prime}} \mathbf{S}_{n n^{\prime}} M_{n n^{\prime}}
\end{aligned}
$$


are the mean-field precession frequencies of the impurity and carrier spins, respectively. The first terms on the right-hand side of Eqs. (4a) and (4b) represent the precession of the impurity and carrier spins in the mean field due to the carrier and impurity magnetization as well as the external magnetic field. The second terms in Eqs. (4a) and (4b) describe the effects of the magnetic carrier-impurity correlations on the impurity and carrier density matrices and the last term of Eq. (4b) describes the scattering of carriers at non-magnetic impurities.

In analogy to the situation without non-magnetic impurity scattering $\left(J_{0}=0\right)$ studied in Ref. 26, we label the source terms of the correlations on the right-hand side of the Eqs. (4c) and (4d) as follows: The terms $b_{\sigma_{1} n_{1} \mathbf{k}_{1}}^{\sigma_{2} n_{2} \mathbf{k}_{2} I}$ are the inhomogeneous driving terms depending only on single-particle quantities. $b_{\sigma_{1} n_{1} \mathbf{k}_{1}}^{\sigma_{2} n_{2} \mathbf{k}_{2} I I}$ are homogeneous terms which describe a precession-type motion of the correlations in the effective fields $\boldsymbol{\omega}_{\mathrm{e}}$ and $\boldsymbol{\omega}_{\mathrm{Mn}}$. The source terms $b_{\sigma_{1} n_{1} \mathbf{k}_{1}}^{\sigma_{2} n_{2} \mathbf{k}_{2}{ }^{I I I}}$ comprise the driving of the magnetic correlations by other magnetic correlations with different wave vectors and describe a change of the wave vectors of the correlations due to the $s$ - $d$ interaction. $b_{\sigma_{1} n_{1} \mathbf{k}_{1}}^{\sigma_{2} n_{2} \mathbf{k}_{2}}$ imp denotes the contributions to the equation for the magnetic correlations due to the non-magnetic impurity scattering. The source terms $c_{\sigma_{1} \mathbf{k}_{1}}^{\sigma_{2} \mathbf{k}_{2} X}$ for the nonmagnetic correlations are classified analogously.

A straightforward but lengthy calculation confirms that Eqs. (4) conserve the particle number as well as the total energy comprised of the single-particle contributions and the correlation energies.

\section{Markov limit}

Although Eqs. (4) can readily be used to calculate the spin dynamics given a set of appropriate initial conditions, it is instructive also to derive the Markov limit of the quantum kinetic equations ${ }^{26-28}$. On the one hand, this enables us to distinguish the Markovian behavior from genuine quantum kinetic effects. On the other hand, it allows us to derive analytic expressions for the correlation energies and the renormalization of the precession frequencies in the presence of an external magnetic field ${ }^{39}$.

The derivation of the Markov limit comprises two steps $^{28}$ : First, the equations of motion for the correlations are formally integrated yielding explicit expressions for the correlations in the form of a memory integral. This yields integro-differential equations for the singleparticle variables, where the values of the single-particle variables at earlier times enter. Second, the memory integral is eliminated by assuming a $\delta$-like short memory.

However, the first step, which involves the formal integration of the carrier-impurity correlations, can, in general, be complicated. Nevertheless, if the source terms $b_{\sigma_{1} n_{1} \mathbf{k}_{1}}^{\sigma_{2} n_{2} \mathbf{k}_{2} I I I}$ and $c_{\sigma_{1} \mathbf{k}_{1}}^{\sigma_{2} \mathbf{k}_{2}}{ }^{I I I}$ as well as the correlation- dependent part of $b_{\sigma_{1} n_{1} \mathbf{k}_{1}}^{\sigma_{2} n_{2} \mathbf{k}_{2} \text { imp }}$ and $c_{\sigma_{1} \mathbf{k}_{1}}^{\sigma_{2} \mathbf{k}_{2}}$ sd are neglected, the formal solution of Eqs. (4c-d) becomes much easier. In absence of non-magnetic impurity scattering, it has been shown that these source terms are indeed numerically insignificant ${ }^{26}$. Furthermore, a straightforward calculation shows that neglecting these terms also yields a consistent theory with respect to the conservation of the total energy. Whether neglecting the terms $b_{\sigma_{1} n_{1} \mathbf{k}_{1}}^{\sigma_{2} n_{2} \mathbf{k}_{2} I I I}, c_{\sigma_{1} \mathbf{k}_{1}}^{\sigma_{2} \mathbf{k}_{2} I I I}$ and the correlation-dependent parts of $b_{\sigma_{1} n_{1} \mathbf{k}_{1}}^{\sigma_{2} n_{2} \mathbf{k}_{2}}$ imp and $c_{\sigma_{1} \mathbf{k}_{1}}^{\sigma_{2} \mathbf{k}_{2}}$ sd is indeed a good approximation in the presence of non-magnetic impurity scattering can be tested by comparing the numerical results of the quantum kinetic equations with and without accounting for these source terms.

Neglecting the aforementioned source terms in Eqs. (4), we first formulate a set of quantum kinetic equations for the new dynamical variables

$$
\begin{aligned}
& \left\langle S^{i}\right\rangle=\sum_{n_{1} n_{2}} S_{n_{1} n_{2}}^{i} M_{n_{1} n_{2}}, \\
& n_{\mathbf{k}}=\sum_{\sigma} C_{\sigma \mathbf{k}}^{\sigma}, \\
& s_{\mathbf{k}}^{i}=\sum_{\sigma_{1} \sigma_{2}} s_{\sigma_{1} \sigma_{2}}^{i} C_{\sigma_{1} \mathbf{k}}^{\sigma_{2}}, \\
& \bar{C}_{\mathbf{k}_{1}}^{\alpha \mathbf{k}_{2}}=\sum_{\sigma_{1} \sigma_{2}} s_{\sigma_{1} \sigma_{2}}^{\alpha} \bar{C}_{\sigma_{1} \mathbf{k}_{1}}^{\sigma_{2} \mathbf{k}_{2}} \\
& Q_{l \mathbf{k}_{1}}^{\alpha \mathbf{k}_{2}}=\sum_{\sigma_{1} \sigma_{2}} \sum_{n_{1} n_{2}} s_{\sigma_{1} \sigma_{2}}^{\alpha} S_{n_{1} n_{2}}^{l} Q_{\sigma_{1} n_{1} \mathbf{k}_{1}}^{\sigma_{2} n_{2} \mathbf{k}_{2}},
\end{aligned}
$$

where $\langle\mathbf{S}\rangle$ is the average impurity spin and $n_{\mathbf{k}}$ and $\mathbf{s}_{\mathbf{k}}$ are the occupation density and spin density of the carrier states with wave vector $\mathbf{k}$, respectively. $\bar{C}_{\mathbf{k}_{1}}^{\alpha \mathbf{k}_{2}}$ as well as $Q_{l \mathbf{k}_{1}}^{\alpha \mathbf{k}_{2}}$ comprise the non-magnetic and magnetic carrier-impurity correlations. In Eqs. (6) we use a notation in which the Latin indices are in the range $\{1,2,3\}$, while the Greek indices also include the value 0 , where $s_{\sigma_{1} \sigma_{2}}^{0}=\delta_{\sigma_{1} \sigma_{2}}$ is the $2 \times 2$ identity matrix. The corresponding equations of motion for the variables defined in Eqs. (6) are explicitly given in appendix A.

Note that the source terms $b_{l \mathbf{k}_{1}}^{\alpha \mathbf{k}_{2} I}$ for the correlations $Q_{l \mathbf{k}_{1}}^{\alpha \mathbf{k}_{2}}$ depend on the second moments of the impurity spins $\left\langle S^{i} S^{j}\right\rangle=\sum_{n_{1} n_{2} n_{3}} S_{n_{1} n_{2}}^{i} S_{n_{2} n_{3}}^{j} M_{n_{1} n_{3}}$ for which we do not present equations of motions, although such equations can, in principle, be derived from Eqs. (4). Here, we use the fact that for typical sample parameters the optically induced carrier density is usually much lower than the impurity concentration, so that the average impurity spin only changes marginally over time ${ }^{26}$. For the numerical calculations we assume that the impurity density matrix can be approximately described as being in thermal equilibrium at all times where the effective impurity spin temperature $T_{\mathrm{Mn}}$ can be obtained from the value of $\langle\mathbf{S}\rangle$. From this thermally occupied density matrix, the second moments $\left\langle S^{i} S^{j}\right\rangle$ consistent with $\langle\mathbf{S}\rangle$ can be calculated in each time step. 
The equations of motion for the variables defined in Eqs. (6) are the starting point for the formal integration of the correlations. Note that Eqs. (A1d-g) for the correlations $Q_{l \mathbf{k}_{1}}^{\alpha \mathbf{k}_{2}}$ and $\bar{C}_{\mathbf{k}_{1}}^{\alpha \mathbf{k}_{2}}$ can be transformed into the general form

$$
\begin{aligned}
\frac{\partial}{\partial t} Q_{\mathbf{k}_{1}}^{\mathbf{k}_{2}} & =-i\left(\omega_{\mathbf{k}_{2}}-\omega_{\mathbf{k}_{1}}\right) Q_{\mathbf{k}_{1}}^{\mathbf{k}_{2}}+i \chi_{1} \omega_{\mathrm{e}} Q_{\mathbf{k}_{1}}^{\mathbf{k}_{2}} \\
& +i \chi_{2} \omega_{M n} Q_{\mathbf{k}_{1}}^{\mathbf{k}_{2}}+b_{\mathbf{k}_{1}}^{\mathbf{k}_{2} I}
\end{aligned}
$$

where $\chi_{1}, \chi_{2} \in\{-1,0,1\}$ and the terms proportional to $\omega_{\mathrm{e}}=\left|\boldsymbol{\omega}_{\mathrm{e}}\right|$ and $\boldsymbol{\omega}_{\mathrm{Mn}}=\left|\boldsymbol{\omega}_{\mathrm{Mn}}\right|$ originate from the precession of the correlations described by the source terms $b_{\sigma_{1} n_{1} \mathbf{k}_{1}}^{\sigma_{2} n_{2} \mathbf{k}_{2} I I}$ and $c_{\sigma_{1} \mathbf{k}_{1}}^{\sigma_{2} \mathbf{k}_{2} I I}$. The term $b_{\mathbf{k}_{1}}^{\mathbf{k}_{2} I}$ here denotes the contributions from the source terms $b_{\sigma_{1} n_{1} \mathbf{k}_{1}}^{\sigma_{2} n_{2} \mathbf{k}_{2} I}, c_{\sigma_{1} \mathbf{k}_{1}}^{\sigma_{2} \mathbf{k}_{2} I}$,

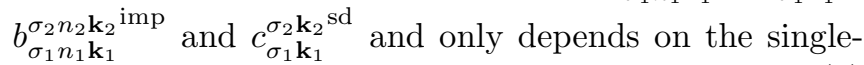
particle variables. The formal integration of Eq. (7) yields

$$
Q_{\mathbf{k}_{1}}^{\mathbf{k}_{2}}(t)=\int_{0}^{t} d t^{\prime} e^{i\left[\omega_{\mathbf{k}_{2}}-\left(\omega_{\mathbf{k}_{1}}+\chi_{1} \omega_{\mathrm{e}}+\chi_{2} \omega_{\mathrm{Mn}}\right)\right]\left(t^{\prime}-t\right)} b_{\mathbf{k}_{1}}^{\mathbf{k}_{2} I}\left(t^{\prime}\right)
$$

The Markov limit consists of assuming a short memory, i.e. the assumption that the correlations at time $t$ depend only significantly on the single-particle variables at the same time $t$, so that one is inclined to evaluate $b_{\mathbf{k}_{1}}^{\mathbf{k}_{2} I}\left(t^{\prime}\right)$ in Eq. (8) at $t^{\prime}=t$ and to draw the source term out of the integral. However, first, one has to make sure that the source terms are indeed slowly changing variables.
For example, the carrier spin can precess rapidly about an external magnetic field. Therefore, we first analyze the mean-field precession of the single-particle quantities and split the source terms into parts oscillating with some frequencies $\omega$ of the form

$$
b_{\mathbf{k}_{1}}^{\mathbf{k}_{2} I}\left(t^{\prime}\right) \stackrel{\mathrm{MF}}{=} \sum_{\omega} \sum_{\chi \in\{-1,0,1\}} e^{i \chi \omega\left(t^{\prime}-t\right)} b_{\mathbf{k}_{1}}^{\mathbf{k}_{2} \omega, \chi}(t) .
$$

Then, the different oscillating parts $b_{\mathbf{k}_{1}}^{\mathbf{k}_{2} \omega, \chi}(t)$ can be drawn out of the memory integral and the remaining integral can be solved in the limit of large times $t^{28}$ :

$$
\int_{0}^{t} d t^{\prime} e^{i \Delta \omega\left(t^{\prime}-t\right)} \stackrel{t \rightarrow \infty}{\longrightarrow} \pi \delta(\Delta \omega)-\frac{i}{\Delta \omega}
$$

This procedure yields particularly transparent results in the case where the external magnetic field and the impurity magnetization are collinear, as is usually the case when the number of impurities exceeds the number of quasi-free carriers $\left(N_{\mathrm{Mn}} \gg N_{e}\right)$, and the impurity density matrix is initially occupied thermally. Choosing the direction of $\boldsymbol{\omega}_{\mathrm{e}}$ as a reference and defining $s_{\mathbf{k}_{1}}^{\|}:=\mathbf{s} \cdot \frac{\boldsymbol{\omega}_{\mathrm{e}}}{\omega_{\mathrm{e}}}$, $S^{\|}:=\hat{\mathbf{S}} \cdot \frac{\boldsymbol{\omega}_{\mathrm{e}}}{\omega_{\mathrm{e}}}$ and $\omega_{\mathrm{Mn}}^{\|}:=\boldsymbol{\omega}_{\mathrm{Mn}} \cdot \frac{\boldsymbol{\omega}_{\mathrm{e}}}{\omega_{\mathrm{e}}}$, the Markovian equations obtained for the spin-up and spin-down occupations and the perpendicular carrier spin component with respect to the direction of $\boldsymbol{\omega}_{\mathrm{e}}$,

$$
\begin{aligned}
n_{\mathbf{k}_{1}}^{\uparrow / \downarrow} & :=\frac{n_{\mathbf{k}_{1}}}{2} \pm s_{\mathbf{k}_{1}}^{\|}, \\
\mathbf{s}_{\mathbf{k}_{1}}^{\perp} & :=\mathbf{s}_{\mathbf{k}_{1}}-\frac{\boldsymbol{\omega}_{\mathrm{e}}}{\omega_{\mathrm{e}}} s_{\mathbf{k}_{1}}^{\|},
\end{aligned}
$$

are given by:

$$
\begin{aligned}
\frac{\partial}{\partial t} n_{\mathbf{k}_{1}}^{\uparrow}= & \frac{\pi}{\hbar^{2} V^{2}} \sum_{\mathbf{k}_{2}}\left\{\delta\left(\omega_{\mathbf{k}_{2}}-\omega_{\mathbf{k}_{1}}\right)\left[J_{\mathrm{sd}}^{2} N_{\mathrm{Mn}} \frac{1}{2}\left\langle S^{\|^{2}}\right\rangle \pm J_{\mathrm{sd}} J_{0}\left(N_{\mathrm{Mn}}+N_{\mathrm{imp}}\right)\left\langle S^{\|}\right\rangle+2 J_{0}^{2} N_{\mathrm{imp}}\right]\left(n_{\mathbf{k}_{2}}^{\uparrow / \downarrow}-n_{\mathbf{k}_{1}}^{\uparrow / \downarrow}\right)+\right. \\
& \left.+\delta\left[\omega_{\mathbf{k}_{2}}-\left(\omega_{\mathbf{k}_{1}} \pm\left(\omega_{\mathrm{e}}-\omega_{\mathrm{Mn}}^{\|}\right)\right)\right] J_{\mathrm{sd}}^{2} N_{\mathrm{Mn}}\left[\left(\left\langle S^{\perp^{2}}\right\rangle \pm \frac{1}{2}\left\langle S^{\|}\right\rangle\right)\left(1-n_{\mathbf{k}_{1}}^{\uparrow / \downarrow}\right) n_{\mathbf{k}_{2}}^{\downarrow / \uparrow}-\left(\left\langle S^{\perp^{2}}\right\rangle \mp \frac{1}{2}\left\langle S^{\|}\right\rangle\right)\left(1-n_{\mathbf{k}_{2}}^{\downarrow / \uparrow}\right) n_{\mathbf{k}_{1}}^{\uparrow / \downarrow}\right]\right\}
\end{aligned}
$$




$$
\begin{aligned}
\frac{\partial}{\partial t} \mathbf{s}_{\mathbf{k}_{1}}^{\perp}= & -\frac{\pi}{\hbar^{2} V^{2}} \sum_{\mathbf{k}_{2}}\left\{\delta\left(\omega_{\mathbf{k}_{2}}-\omega_{\mathbf{k}_{1}}\right)\left[J_{\mathrm{sd}}^{2} N_{\mathrm{Mn}} \frac{1}{2}\left\langle S^{\|^{2}}\right\rangle\left(\mathbf{s}_{\mathbf{k}_{2}}^{\perp}+\mathbf{s}_{\mathbf{k}_{1}}^{\perp}\right)-2 J_{0}^{2} N_{\mathrm{imp}}\left(\mathbf{s}_{\mathbf{k}_{2}}^{\perp}-\mathbf{s}_{\mathbf{k}_{1}}^{\perp}\right)\right]\right. \\
& +\delta\left[\omega_{\mathbf{k}_{2}}-\left(\omega_{\mathbf{k}_{1}}+\left(\omega_{\mathrm{e}}-\omega_{\mathrm{Mn}}^{\|}\right)\right)\right] \frac{1}{2}\left(\left\langle S^{\perp^{2}}\right\rangle-\frac{1}{2}\left\langle S^{\|}\right\rangle\left(1-2 n_{\mathbf{k}_{2}}^{\perp}\right)\right) \mathbf{s}_{\mathbf{k}_{1}}^{\perp} \\
& +\delta\left[\omega_{\mathbf{k}_{2}}-\left(\omega_{\mathbf{k}_{1}}-\left(\omega_{\mathrm{e}}-\omega_{\mathrm{Mn}}^{\|}\right)\right) \frac{1}{2}\left(\left\langle S^{\perp}{ }^{2}\right\rangle+\frac{1}{2}\left\langle S^{\|}\right\rangle\left(1-2 n_{\mathbf{k}_{2}}^{\uparrow}\right)\right) \mathbf{s}_{\mathbf{k}_{1}}^{\perp}\right\} \\
& +\boldsymbol{\omega}_{\mathrm{e}} \times \mathbf{s}_{\mathbf{k}_{1}}^{\perp}+\frac{1}{\hbar^{2} V^{2}} \sum_{\mathbf{k}_{2}}\left\{-\frac{J_{\mathrm{sd}} J_{0}}{\omega_{\mathbf{k}_{2}}-\omega_{\mathbf{k}_{1}}}\langle\mathbf{S}\rangle \times\left[\left(N_{\mathrm{imp}}-N_{\mathrm{Mn}}\right) \mathbf{s}_{\mathbf{k}_{2}}^{\perp}+\left(N_{\mathrm{Mn}}+N_{\mathrm{imp}}\right) \mathbf{s}_{\mathbf{k}_{1}}^{\perp}\right]\right. \\
& -\frac{J_{\mathrm{sd}}^{2} N_{\mathrm{Mn}}}{\omega_{\mathbf{k}_{2}}-\left(\omega_{\mathbf{k}_{1}}+\left(\omega_{\mathrm{e}}-\omega_{\mathrm{Mn}}^{\|}\right)\right)} \frac{1}{2}\left(\left\langle S^{\perp^{2}}\right\rangle-\frac{1}{2}\left\langle S^{\|}\right\rangle\left(1-2 n_{\mathbf{k}_{2}}^{\downarrow}\right)\right)\left(\frac{\boldsymbol{\omega}_{\mathrm{e}}}{\omega_{\mathrm{e}}} \times \mathbf{s}_{\mathbf{k}_{1}}^{\perp}\right) \\
& \left.+\frac{J_{\mathrm{sd}}^{2} N_{\mathrm{Mn}}}{\omega_{\mathbf{k}_{2}}-\left(\omega_{\mathbf{k}_{1}}-\left(\omega_{\mathrm{e}}-\omega_{\mathrm{Mn}}^{\|}\right)\right)} \frac{1}{2}\left(\left\langle S^{\perp}\right\rangle+\frac{1}{2}\left\langle S^{\|}\right\rangle\left(1-2 n_{\mathbf{k}_{2}}^{\uparrow}\right)\right)\left(\frac{\boldsymbol{\omega}_{\mathrm{e}}}{\omega_{\mathrm{e}}} \times \mathbf{s}_{\mathbf{k}_{1}}^{\perp}\right)\right\} .
\end{aligned}
$$

The first line of the right-hand side of Eq. (12a), which is proportional to $n_{\mathbf{k}_{2}}^{\uparrow / \downarrow}-n_{\mathbf{k}_{1}}^{\uparrow / \downarrow}$, describes a redistribution of occupations of spin-up and spin-down states within a shell of defined kinetic energy via the term proportional to $\delta\left(\omega_{\mathbf{k}_{2}}-\omega_{\mathbf{k}_{1}}\right)$. For a parabolic band structure, this implies a redistribution between states with the same modulus $k$ of the wave vector $\mathbf{k}$, while the total carrier spin remains unchanged. If accompanied by a wave-vector dependent magnetic field like a Rashba or the Dresselhaus field, this term leads to a D'yakonovPerel'-type suppression of the spin dephasing. Here, however, we do not consider any wave vector dependent field and the system under investigation is isotropic in $\mathbf{k}$-space, so that the first line in Eq. (12a) has no influence on the dynamics of the total spin. The second line in Eq. (12a) describes a spin-flip scattering from the spin-up band to the spin-down band and vice versa. Since these bands are energetically split by $\hbar \omega_{\mathrm{e}}$ and a flip of carrier spin involves a corresponding flip of an impurity spin in the opposite direction, which requires a magnetic (Zeeman) energy of $\hbar \omega_{\mathrm{Mn}}^{\|}$, the total magnetic energy released in a spin-flip process is $\pm \hbar\left(\omega_{\mathrm{e}}-\omega_{\mathrm{Mn}}^{\|}\right)$. Thus, $\delta\left[\omega_{\mathbf{k}_{2}}-\left(\omega_{\mathbf{k}_{1}} \pm\left(\omega_{\mathrm{e}}-\omega_{\mathrm{Mn}} \|\right)\right]\right.$ ensures a conservation of the total single-particle energies in the Markov limit. It is noteworthy that, if the mean-field dynamics of the source terms as in Eq. (9) is not correctly taken into account, other energetic shifts are obtained in the $\delta$ function, which yields equations in the Markov limit that are not consistent with the conservation of the singleparticle energies ${ }^{28}$. Note also that the right-hand side of Eq. (12a) correctly deals with Pauli blocking effects. Because the non-magnetic impurity scattering enters in the equations of motion (12a) for the spin-up and spin-down occupation only via the first line which plays no role in an isotropic system, it has no influence on the spin dynamics in the Markov limit.

The first three lines in Eq. (12b) for the perpendicular carrier spin component, which are proportional to $\delta$-functions, indicate an exponential decay of the per- pendicular carrier spin component towards zero. The last three lines describe a precession of the perpendicular carrier spin component. The mean-field precession frequency $\omega_{\mathrm{e}}$ is renormalized by the carrier-impurity correlations. This renormalization originates from the imaginary part of the memory integral in Eq. (10). Besides the terms proportional to $\frac{1}{\omega_{\mathbf{k}_{2}}-\left(\omega_{\mathbf{k}_{1}} \pm\left(\omega_{\mathrm{e}}-\omega_{\mathrm{Mn}_{n}}^{\|}\right)\right)}$, which are also present when only the magnetic $s-d$ interaction is taken into account ${ }^{39}$, the non-magnetic impurity scattering introduces another contribution which is a cross-term, i.e. it is absent when either the magnetic or the nonmagnetic impurity scattering is absent, which can be seen from the fact that it is proportional to the product of $J_{\mathrm{sd}}$ and $J_{0}$. In the quasi-continuous limit, the sum over $\mathbf{k}_{2}$ can be replaced by an integral over the spectral density of states. In quasi-two-dimensional systems like quantum wells, the spectral denstiy of states $D(\omega)=\frac{A m^{*}}{2 \pi \hbar}$ is constant. Thus, the frequency renormalization can be integrated and yields logarithmic divergences

$$
\begin{aligned}
& \sum_{\mathbf{k}_{2}} \frac{1}{\omega_{\mathbf{k}_{2}}-\omega_{0}}=\int_{0}^{\omega_{B Z}} d \omega D(\omega) \frac{1}{\omega-\omega_{0}} \\
& =\frac{A m^{*}}{2 \pi \hbar} \ln \left|\frac{\omega_{B Z}-\omega_{0}}{\omega_{0}}\right| .
\end{aligned}
$$

at the poles $\omega_{0}=\omega_{\mathbf{k}_{1}}$ and $\omega_{0}=\omega_{\mathbf{k}_{1}} \pm\left(\omega_{\mathrm{e}}-\omega_{\mathrm{Mn}}^{\|}\right)$. These logarithmic divergences are similar to the ones obtained in the discussion of the Kondo-effect in metals with magnetic impurities ${ }^{29}$. Despite the formal divergence, the summation over a non-singular carrier distribution always leads to a finite value of the precession frequency of the total carrier spin, since the logarithm is integrable ${ }^{28}$. From Eq. (13), one can see that the cut-off energy $\hbar \omega_{B Z}$, which corresponds to the width of the conduction band and is typically of the order of $1 \mathrm{eV}$, enters as a new model parameter in the theory and cannot be eliminated by assuming that $\omega_{B Z} \rightarrow \infty$, since then the frequency renormalization also diverges. As a consequence, the Markovian expression for the frequency renormalization 
can only give an order-of-magnitude estimation and a more detailed treatment of the band structure is necessary if a quantitatively more accurate description is required.

For the special case of zero external magnetic field, vanishing impurity magnetization and low carrier densities, Eqs. (12) are equivalent to the simple rate equations

$$
\frac{\partial}{\partial t} \mathbf{S}_{\mathbf{k}_{1}}=-\frac{1}{\tau} \mathbf{S}_{\mathbf{k}_{1}}
$$

where the values of the rates coincide with the Fermi's golden rule value. In two dimensions, one obtains ${ }^{25}$

$$
\frac{1}{\tau^{2 D}}=\frac{35}{12} \frac{J_{\mathrm{sd}}^{2} m^{*}}{\hbar^{3}} \frac{N_{\mathrm{Mn}}}{V} \frac{1}{d}
$$

\section{Correlation energy}

In Eqs. (8) to (10), Markovian expressions for the carrier-impurity correlations are derived as functionals of the carrier and impurity variables. Using these expressions, it is straightforward to also obtain analytic expressions for the carrier-impurity correlation energies as functionals of the carrier spins and occupations ${ }^{28}$. Splitting the averages over the magnetic and non-magnetic carrier-impurity interactions into mean-field and correlated contributions

$$
\begin{aligned}
\left\langle H_{\mathrm{sd}}\right\rangle & =\left\langle H_{\mathrm{sd}}^{\mathrm{MF}}\right\rangle+\left\langle H_{\mathrm{sd}}^{\mathrm{cor}}\right\rangle, \\
\left\langle H_{\mathrm{imp}}\right\rangle & =\left\langle H_{\mathrm{imp}}^{\mathrm{MF}}\right\rangle+\left\langle H_{\mathrm{imp}}^{\mathrm{cor}}\right\rangle, \\
\left\langle H_{\mathrm{sd}}^{\mathrm{MF}}\right\rangle & =\frac{J_{\mathrm{sd}} N_{\mathrm{Mn}}}{V} \sum_{\mathbf{k}}\langle\mathbf{S}\rangle \cdot \mathbf{s}_{\mathbf{k}} \\
\left\langle H_{\mathrm{sd}}^{\mathrm{cor}}\right\rangle & =\frac{J_{\mathrm{sd}} N_{\mathrm{Mn}}}{V^{2}} \sum_{\mathbf{k}, \mathbf{k}^{\prime}} \sum_{i} Q_{i \mathbf{k}}^{i \mathbf{k}^{\prime}} \\
\left\langle H_{\mathrm{imp}}^{\mathrm{MF}}\right\rangle & =\frac{J_{0} N_{\mathrm{imp}}}{V} \sum_{\mathbf{k}} n_{\mathbf{k}}, \\
\left\langle H_{\mathrm{imp}}^{\mathrm{cor}}\right\rangle & =\frac{J_{0} N_{\mathrm{imp}}}{V^{2}} \sum_{\mathbf{k}, \mathbf{k}^{\prime}} \bar{C}_{\mathbf{k}}^{0 \mathbf{k}^{\prime}},
\end{aligned}
$$

one obtains in the Markov limit

$$
\begin{aligned}
\left\langle H_{\mathrm{sd}}^{\mathrm{cor}}\right\rangle= & -\frac{J_{\mathrm{sd}} N_{\mathrm{Mn}}}{V^{2}} \sum_{\mathbf{k}_{1} \mathbf{k}_{2}}\left\{\frac{\frac{1}{2} J_{\mathrm{sd}}\left\langle S^{\|^{2}}\right\rangle n_{\mathbf{k}_{1}}+2 J_{0}\left\langle S^{\|}\right\rangle s_{\mathbf{k}_{1}}^{\|}}{\omega_{\mathbf{k}_{2}}-\omega_{\mathbf{k}_{1}}}\right. \\
& +\frac{J_{\mathrm{sd}}\left(\left\langle S^{\perp}\right\rangle-\frac{1}{2}\left\langle S^{\|}\right\rangle\right)\left(1-n_{\mathbf{k}_{2}}^{\downarrow}\right) n_{\mathbf{k}_{1}}^{\uparrow}}{\omega_{\mathbf{k}_{2}}-\left(\omega_{\mathbf{k}_{1}}+\left(\omega_{\mathrm{e}}-\omega_{\mathrm{Mn}}^{\|}\right)\right)} \\
& \left.+\frac{J_{\mathrm{sd}}\left(\left\langle S^{\perp}\right\rangle+\frac{1}{2}\left\langle S^{\|}\right\rangle\right)\left(1-n_{\mathbf{k}_{2}}^{\uparrow} n_{\mathbf{k}_{1}}^{\downarrow}\right.}{\omega_{\mathbf{k}_{2}}-\left(\omega_{\mathbf{k}_{1}}-\left(\omega_{\mathrm{e}}-\omega_{\mathrm{Mn}}^{\|}\right)\right)}\right\}, \quad(17 \mathrm{a}) \\
\left\langle H_{\mathrm{imp}}^{\mathrm{cor}}\right\rangle= & -2 \frac{J_{0} N_{\mathrm{imp}}}{V^{2}} \sum_{\mathbf{k}_{1} \mathbf{k}_{2}} \frac{J_{0} n_{\mathbf{k}_{1}}+J_{\mathrm{sd}}\left\langle S^{\|}\right\rangle s_{\mathbf{k}_{1}}^{\|}}{\omega_{\mathbf{k}_{2}}-\omega_{\mathbf{k}_{1}}} .
\end{aligned}
$$

Eqs. (17) have the same poles as Eq. (12b) for the frequency renormalization and, thus, also contain formally logarithmic divergences in two-dimensional systems.

\section{RESULTS}

After having derived the quantum kinetic equations for the description of the spin dynamics in DMS including magnetic and non-magnetic scattering and having obtained rate-type Markovian equations, we now present results of numerical simulations. Here, we focus on the case of a 4 -nm-wide $\mathrm{Cd}_{0.93} \mathrm{Mn}_{0.07} \mathrm{Te}$ quantum well. For this material, the magnetic coupling constant is $J_{\mathrm{sd}}=$ $-15 \mathrm{meVnm}^{3}\left(N_{0} J_{\mathrm{sd}}=-220 \mathrm{meV}\right)^{42}$, while the nonmagnetic coupling constant is approximately $J_{0}=110$ $\mathrm{meVnm}^{3}\left(N_{0} J_{0}=1.6 \mathrm{eV}\right)^{31}$, where $N_{0}$ is the number of unit cells per unit volume. Furthermore, we use a conduction band effective mass of $m^{*}=0.1 m_{0}$ and assume that the impurity magnetization is described by a thermal distribution at a temperature of $T=2 \mathrm{~K}$ and the $\mathrm{g}$-factors of the conduction band carriers and $\mathrm{Mn}$ impurities are $g_{e}=-1.77$ and $g_{\mathrm{Mn}}=2$, respectively ${ }^{40}$. If not stated otherwise, we choose a value of $40 \mathrm{meV}$ for the cut-off energy $\hbar \omega_{B Z}$ in the numerical calculations and we consider only $\mathrm{Mn}$ ions as sources of non-magnetic impurity scattering, i. e. $N_{\mathrm{imp}}=N_{\mathrm{Mn}}$. As initial value for the carrier distribution, we use a Gaussian distribution centered at the band edge of the spin-up band with standard deviation of $E_{s}=0.4 \mathrm{meV}$, which corresponds to an excitation with a circularly polarized light pulse with full width at half maximum (FWHM) pulse duration of about 350 fs.

We first discuss the spin dynamics in the conduction band of a $\mathrm{Cd}_{0.93} \mathrm{Mn}_{0.07} \mathrm{Te}$ quantum well for zero magnetic field with a focus on the impact of non-magnetic impurity scattering on the spin dynamics and investigate the redistribution of carriers in $\mathbf{k}$-space as well as the build-up of correlation energy. Then, we study the spin dynamics in the valence band in a simplified model. Finally, we investigate the spin dynamics in the presence of an external magnetic field parallel and perpendicular to the carrier spin polarization and discuss, in the latter case, how the non-magnetic impurity scattering affects the carrier spin precession frequencies.

\section{A. Zero magnetic field}

Figure 1(a) shows the time evolution of an initially polarized carrier spin in a $\mathrm{Cd}_{0.93} \mathrm{Mn}_{0.07} \mathrm{Te}$ quantum well for vanishing magnetic field. The Markovian equations (12) predict a simple exponential decay of the carrier spin, which is transferred to the impurities. Note that due to $N_{\mathrm{Mn}} \gg N_{e}$, the asymptotic value of the carrier spin for long times $t$ is close to zero, since the impurities act as a spin bath. If only the magnetic spinflip scattering is accounted for $\left(J_{0}=0\right)$, the time evolution according to the quantum kinetic theory is nonmonotonic and shows an overshoot below the asymptotic value. These non-Markovian effects are strongly suppressed in the calculations including non-magnetic impurity scattering $\left(J_{0}=110 \mathrm{meVnm}^{3}\right)$ and the time evo- 



FIG. 1. (a): Time evolution of the carrier spin for zero magnetic field with $\left(J=110 \mathrm{meVnm}^{3}\right)$ and without $(J=0)$ nonmagnetic impurity scattering. QKT1 (points) denotes the results according to the full quantum kinetic equations (4) while QKT2 (lines) describes the results of the reduced set of equations (A1). The purple dash-dotted line shows the results of the Markovian equations (12), which is independent of non-magnetic impurity scattering. The inset shows a magnification of the region where the quantum kinetic theory for $J_{0}=0$ predicts a non-monotonic behavior. (b) Occupation of carrier states at $t=0$ and $t=10 \mathrm{ps}$ for the calculations with and without non-magnetic impurity scattering.

lution of the total spin follows the Markovian dynamics more closely. An exponential fit to the dynamics of the full quantum kinetic theory yields an effective spin transfer rate about $15 \%$ smaller than the Markovian rate in Eq. (15).

Interestingly, while the full quantum kinetic equations (4) yield identical results as the reduced set of equations (A1) in the case without non-magnetic impurity scattering, deviations between both approaches can be clearly seen when the non-magnetic impurity scattering is taken into account.

In order to understand the suppression of the nonmonotonic features in the spin dynamics with nonmagnetic impurity scattering, it is useful to recapitulate the findings of Ref. 38, where the origin of the nonMarkovian behavior of the spin dynamics in absence of non-magnetic impurity scattering was discussed: It was found that the depth of the memory induced by the correlations is of the order of the inverse energetic distance of the carrier state under consideration to the band edge times $\hbar$. Memory effects become insignificant if the kinetic energy of the carrier $\hbar \omega_{\mathbf{k}_{1}}$ is much higher than the energy scale of the carrier-impurity spin transfer rate $\frac{\hbar}{\tau}$. For the parameters used in the simulations, one obtains from Eq. (15) a value of $\tau^{2 D}=2.97 \mathrm{ps}$ and therefore $\frac{\hbar}{\tau} \approx 0.22 \mathrm{meV}$. Figure $1(\mathrm{~b})$ shows the redistribu- tion of carriers in the calculations with and without nonmagnetic impurity scattering. One can clearly see that, while without non-magnetic impurity scattering the carrier distribution at $t=10 \mathrm{ps}$ is only slightly broadened, including the non-magnetic impurity scatterings leads to a drastic redistribution of carriers to states many meV away from the initial distribution. For these states, the memory is very short compared with the spin relaxation time and therefore the Markovian approximation is justified.

The redistribution of carriers to states several meV away from the band edge raises questions about the conservation of energy, since for zero magnetic field the mean-field energy of the system is comprised of only the kinetic energy of the carriers. In the quantum kinetic calculations, however, we also consider the carrier-impurity correlations which introduce correlation energies that are not captured in a simple single-particle picture. The different contributions to the total energy over the course of time for the simulations presented in Fig. 1 are shown in Fig. 2. There, it is shown that the average kinetic energy per electron increases from the initial value of the order of the width of the initial carrier distribution to a much larger value of about $4 \mathrm{meV}$ on a timescale of about 0.5 ps. This energy is mostly provided by a decrease of nonmagnetic correlation energy from zero to a negative value. 


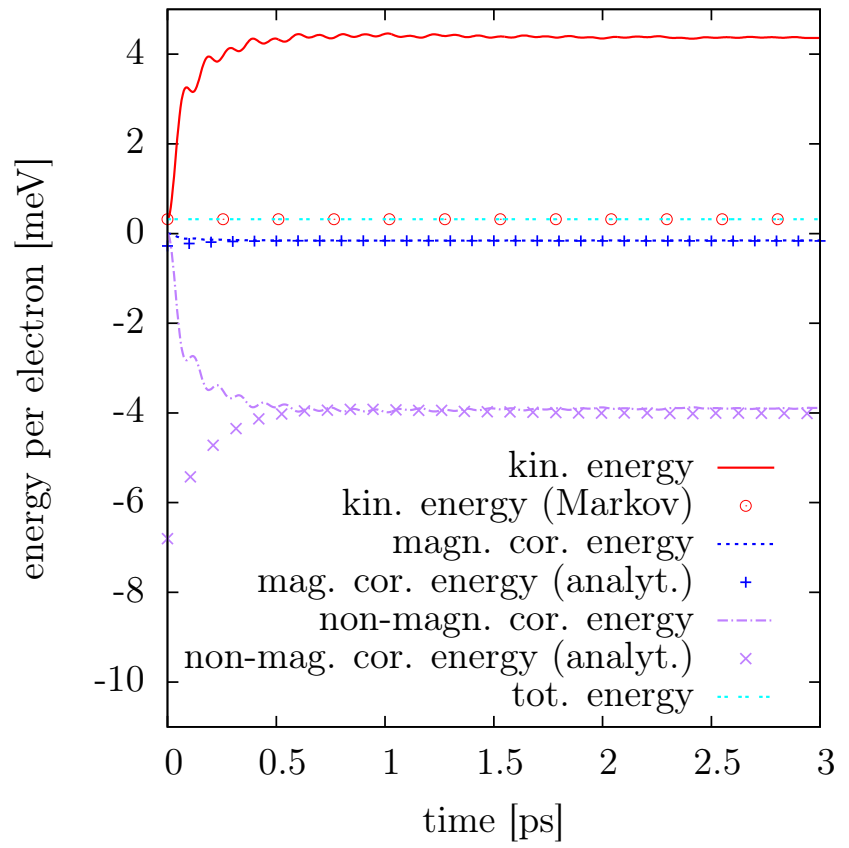

FIG. 2. Kinetic energy (red line), magnetic correlation energy (blue line), non-magnetic correlation energy (purple line) and total energy (cyan line) per electron for the quantum kinetic calculation shown in Fig. 1 with $J_{0}=110 \mathrm{meVnm}^{3}$. The red circles show the kinetic energy obtained from the Markovian calculation in Fig. 1. The pluses and crosses depict the results according to the analytic Markovian expressions for the correlation energies in Eqs. (17) evaluated using the carrier distribution of the quantum kinetic calculation at selected time steps.

The magnetic correlation energy is comparatively small since the magnetic coupling constant $J_{\mathrm{sd}}$ is about one order of magnitude smaller than the non-magnetic coupling constant $J_{0}$. The pluses and crosses in Fig. 2 show the results of the analytic expressions (17) for the correlation energies evaluated using the carrier distributions of the full quantum kinetic theory in the respective time steps. The analytic results are found to coincide with the values extracted from the quantum kinetic theory after the first $0.5 \mathrm{ps}$. Even though the analytic expressions for the correlation energies are obtained within the Markovian description, it should be noted that in the Markovian equations of motion (12) for the spins and occupations only single-particle energies are considered for evaluating the energy balance. As in our case the single particle energies comprise only the kinetic energies of the carriers, the latter are constant in the Markovian description in sharp contrast to the quantum kinetic treatment.

Note also that the total energy comprised of singleparticle and correlation energies remains constant in the quantum kinetic simulations, which provides a further test for the numerics.

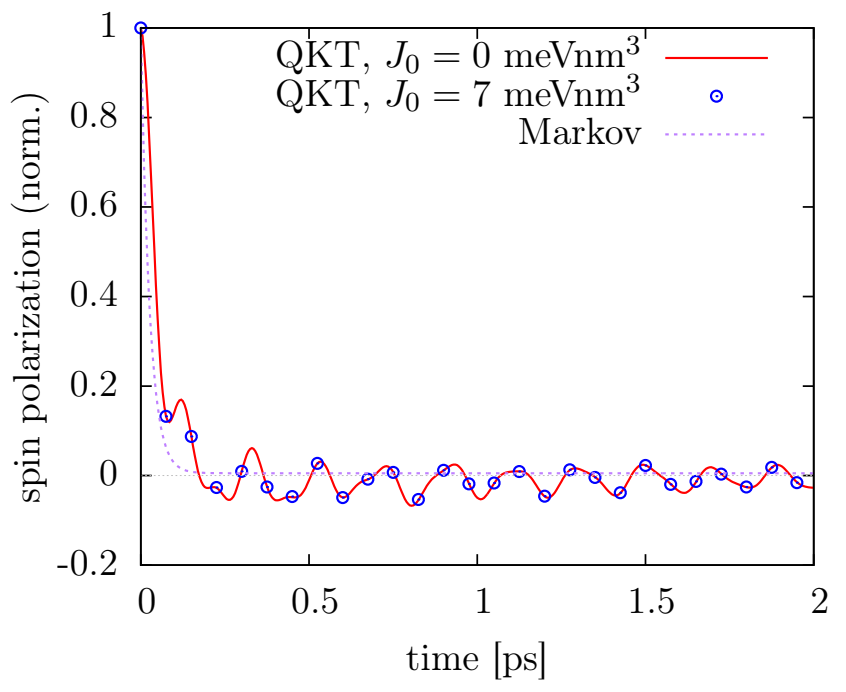

FIG. 3. Spin dynamics in a degenerate valence band of a $\mathrm{Cd}_{0.93} \mathrm{Mn}_{0.07} \mathrm{Te}$ quantum well with and without accounting for non-magnetic impurity scattering.

\section{B. Valence band}

The fact that in the conduction band of a $\mathrm{Cd}_{1-x} \mathrm{Mn}_{x} \mathrm{Te}$ quantum well the non-magnetic scattering at the impurities suppresses the characteristic non-monotonic features of genuine quantum kinetic behavior raises the question whether this statement is true in general and nonMarkovian effects always only change the spin dynamics quantitatively. In this section, we provide an example of a situation where the non-Markovian features are not suppressed due to impurity scattering.

We consider now the valence band of a $\mathrm{Cd}_{1-x} \mathrm{Mn}_{x} \mathrm{Te}$ quantum well. The details of the valence band structure in a quantum well are influenced by, e.g., spin-orbit coupling, strain or the shape of the confinement potential. A realistic description of the band structure is beyond the scope of this article. Instead, we perform a model study, where we assume that heavy-hole and light-hole bands are degenerate. In this case, we can use the quantum kinetic theory derived for the conduction band and take the material parameters for the heavy holes. The magnetic coupling constant in the valence band is $J_{\mathrm{pd}}=60$ $\mathrm{meVnm}^{342}$ and the heavy-hole mass is $m_{h}=0.7 m_{0}{ }^{43}$. The difference of the band gaps between CdTe and zincblende $\mathrm{MnTe}$ of about $1.6 \mathrm{eV}$ is split into the conduction and valence band offsets by a ratio of $14: 1^{44}$. Thus, one obtains a value for the non-magnetic coupling constant in the valence band of about $J_{0}=7 \mathrm{meVnm}^{3}$. The results of the quantum kinetic simulations for these parameters are shown in Fig. 3.

In comparison with the conduction band, the 4 times larger magnetic coupling constant in the valence band leads to much stronger non-Markovian effects. In particular, one finds not a single overshoot, but pronounced oscillations of the spin polarization about its asymptotic 

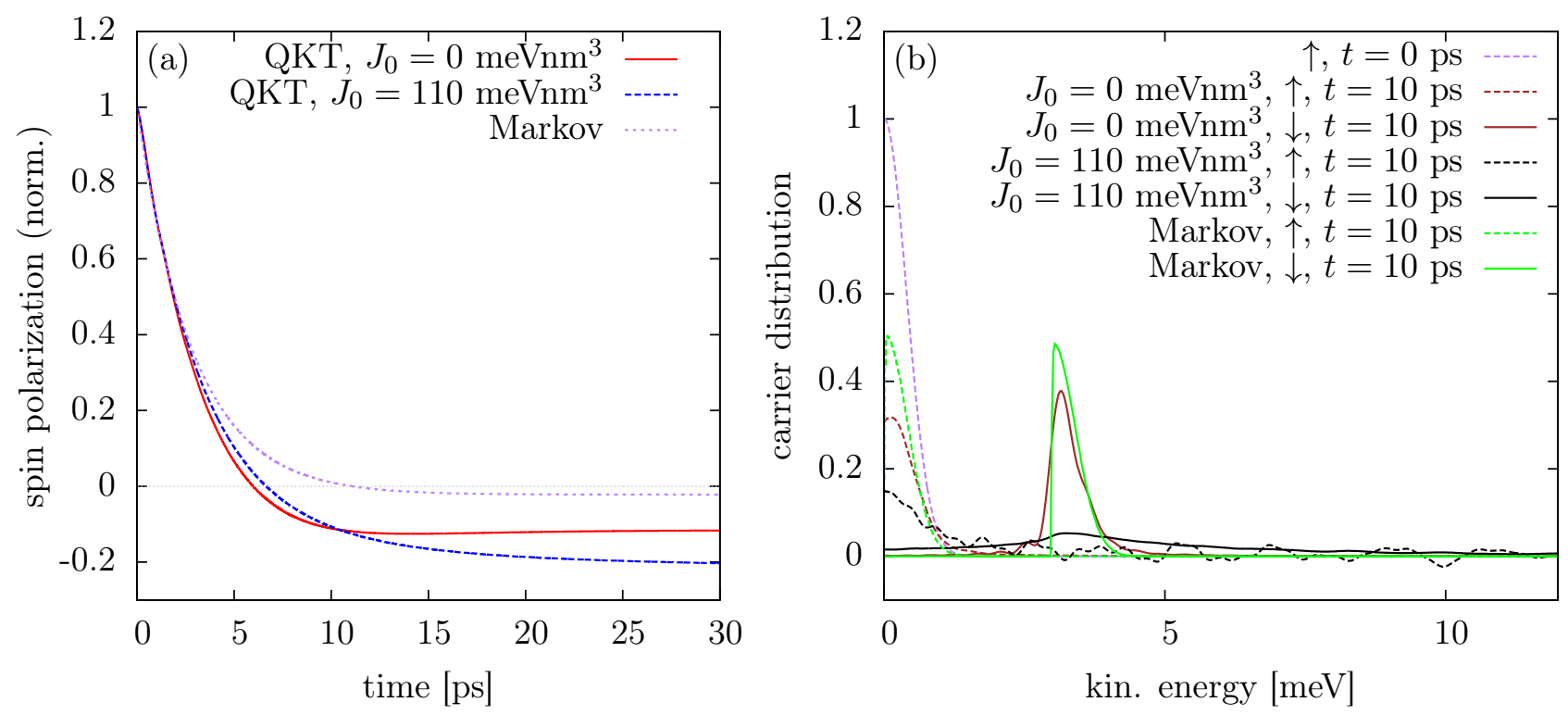

FIG. 4. (a): Time evolution of the carrier spin polarization parallel to an external magnetic field ( $B=100 \mathrm{mT})$. (b): Spin-up $(\uparrow)$ and spin-down occupations $(\downarrow)$ at $t=0$ and $t=10 \mathrm{ps}$.

value. In Fig. 3, the calculations with and without accounting for non-magnetic impurity scattering yield practically identical results. Thus, due to the fact that in the valence band the non-magnetic coupling constant is much smaller than the magnetic coupling constant, no suppression of non-Markovian effects in the spin dynamics is observed.

\section{Finite magnetic field: Faraday configuration}

Next, we investigate the effects of non-magnetic impurity scattering on the spin dynamics in DMS in the presence of an external magnetic field. In this section, we study the case in which the external field and the initial carrier spins are parallel, which is known as the Faraday configuration. This case has also been considered in Ref. 40, but without accounting for non-magnetic impurity scattering.

In Fig. 4(a) the time evolution of the carrier spin polarization parallel to an external magnetic field $B=100$ $\mathrm{mT}$ is shown. Note that the non-monotonic behavior that can be seen in the case without an external magnetic field is suppressed for finite external fields even if the non-magnetic scattering is disregarded. The most striking feature in the time evolution of the carrier spin polarization is that the Markovian result and the quantum kinetic simulations predict very different asymptotic values of the spin polarization at long times $t$.

As discussed in Ref. 40, the different stationary values are related to a broadening of the distribution of scattered carriers in the spin-down band, which is shown in Fig. 4(b). Note that the broadening of the carrier distribution is not primarily an effect of energy-time un- certainty, which is commonly found in quantum kinetic studies $^{45,46}$, since the width of the distribution does not shrink significantly over the course of time ${ }^{40}$. Rather, it is a consequence of the build-up of correlation energy which enables deviations from the conservation of the single-particle energies in spin-flip scattering processes.

In the Markov limit, the stationary value is obtained when a balance between scattering from the spin-up to the spin-down band and vice versa is reached. In the quantum kinetic calculations, the distribution of the scattered carriers is broadened, so that also spin-down states below the threshold $\hbar \omega_{\mathrm{e}}-\hbar \omega_{\mathrm{Mn}}^{\|}$are occupied, whose back-scattering is suppressed since there are no states in the spin-up band with the matching single-particle energies. If additionally the non-magnetic impurity scattering is taken into account, the scattered impurity distribution is even broader and more spin-down states with kinetic energies below $\hbar \omega_{\mathrm{e}}-\hbar \omega_{\mathrm{Mn}}^{\|}$are occupied, so that the back-scattering is more strongly suppressed and the deviation of the asymptotic value of the spin polarization from the Markovian value is even larger.

\section{Finite magnetic field: Voigt configuration}

The situation in which an external magnetic field and the optically induced carrier spin polarization are perpendicular to each other is usually referred to as the Voigt configuration and is the subject of this section. In this situation, the carrier spin precesses about the effective magnetic field $\boldsymbol{\omega}_{\mathrm{e}}$ due to the external field and the impurity magnetization. As shown in Ref. 39, where the non-magnetic impurity scattering was disregarded, the carrier-impurity correlations are responsible for a renor- 

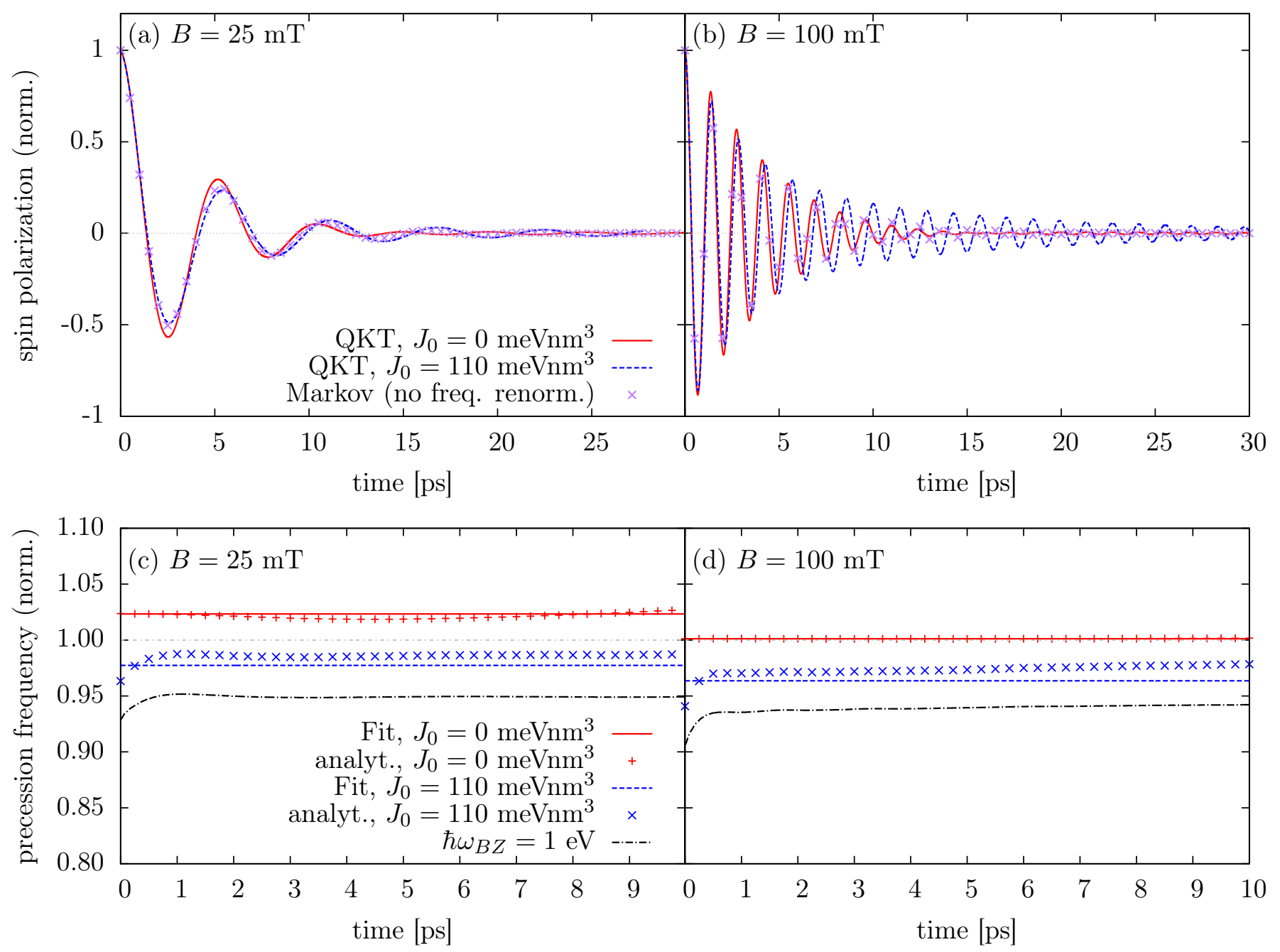

FIG. 5. (a) and (b): Time evolution of the carrier spin polarization for $B=25 \mathrm{mT}$ (a) and $B=100 \mathrm{mT}$ (b) using the quantum kinetic equations (4) and the Markovian equations Eq. (12b), where the terms responsible for the frequency renormalization in the Markovian equations have been dropped. The precession frequency normalized with respect to its mean-field value $\omega_{\mathrm{e}}$ is shown in (c) and (d) using a fit of an exponentially decaying cosine to the quantum kinetic results and the analytic expressions obtained from Eq. (12b) and the occupations from the quantum kinetic calculations. The black dash-dotted lines in (c) and (d) show the analytic results for a cut-off energy of $\hbar \omega_{B Z}=1 \mathrm{eV}$.

malization of the precession frequency. There, it was also shown that the strength of this renormalization depends on the details of the carrier distribution and the strength of the effective field $\boldsymbol{\omega}_{\mathrm{e}}$.

In Fig. 5(a), we present simulations of the spin dynamics in a DMS in Voigt geometry for an external magnetic field of $B=25 \mathrm{mT}$, which corresponds to a situation with $|\langle\mathbf{S}\rangle| \approx 0.05$, where the magnetic-correlation-induced frequency renormalization according to Ref. 39 is particularly strong. Simulations with $\left(J_{0}=110 \mathrm{meVnm}^{3}\right)$ and without $\left(J_{0}=0\right)$ accounting for the non-magnetic impurity scattering are compared to Markovian calculations based on Eqs. (12). Note that for the Markovian calculation shown in Fig. 5 the frequency renormalization was not taken into account. The results of all simulations shown in Fig. 5(a) are very similar and follow closely the form of an exponentially damped cosine. Note that at long times, the phases of the oscillations of the calculations accounting for non-magnetic impurity scattering matches the Markovian calculation without frequency renormalization, while accounting only for magnetic spinflip scattering leads to oscillations with a slightly higher frequency.

The frequency renormalization for the simulations shown in Fig. 5(a) is presented in Fig. 5(c), where an exponentially decaying cosine is fit to the quantum kinetic results and, for comparison, the total precession frequency including the correlation-induced renormalization in the Markovian description in Eq. (12b) evaluated using the spin-up and spin-down occupations of the quantum kinetic simulations is depicted. Due to the time evolution of the occupations, also the renormalization predicted by Eq. (12b) becomes a function of time, which, however, is for all times close to the constant extracted 
by fitting the quantum kinetic result. The calculations without non-magnetic impurity scattering predict an increase of the carrier spin precession frequency of about $2-3 \%$ with respect to the mean-field value $\omega_{\mathrm{e}}$, which is consistent with the findings of Ref. 39. On the other hand, the contribution from the non-magnetic carrierimpurity correlations leads to a decrease of the precession frequency which partially cancels the contribution from the magnetic correlations.

In Figs. 5(b) and 5(d), the time evolution of the carrier spin polarization and the frequency renormalization are shown for an external magnetic field of $B=100$ $\mathrm{mT}$. In this case, the envelope of the spin polarization decays only exponentially for the calculations without non-magnetic impurity scattering. For $J_{0}=110$ $\mathrm{meVnm}^{3}$, the spin polarization follows the exponential decay of the simulation with $J_{0}=0$ only up to about 5 ps. After that, it decays much slower, which is a new non-Markovian effect that is absent if the non-magnetic impurity scattering is disregarded. As can be seen in Fig. 5(d), the frequency renormalization due to the magnetic interaction alone is almost zero. Nevertheless, when the non-magnetic carrier-impurity correlations are taken into account, the precession frequency shows a decrease of about $2-3 \%$. Thus, in contrast to the correlationinduced renormalization in absence of non-magnetic scattering where the renormalization is only observable for a very narrow set of initial conditions ${ }^{39}$, including the nonmagnetic carrier-impurity interaction results in a significant renormalization for a much broader set of excitation conditions.

It is noteworthy that the frequency renormalization in the quantum kinetic calculations is well reproduced by the Markovian expression in Eq. (12b). The numerical demands of the full quantum kinetic equations require a restriction of the conduction band width $\hbar \omega_{B Z}$ used in the calculations to a few tens of meV. However, in realistic band structures, the band widths are of the order of $\mathrm{eV}$. In order to give an order-of-magnitude estimation of the frequency renormalization for such band widths, we present in Figs. 5(c) and 5(d) also the results of the Markovian expression for the frequency renormalizations using the value of $\hbar \omega_{B Z}=1 \mathrm{eV}$ together with the occupations obtained in the quantum kinetic calculations for $\hbar \omega_{B Z}=40 \mathrm{meV}$. This estimation yields a renormalization of the precession frequencies due to the combined effects of magnetic and non-magnetic scattering of about $5-7 \%$. A quantitatively more accurate description requires a more detailed treatment of the band structure, which is beyond the scope of this article.

Note also that the frequency renormalization due to the non-magnetic carrier-impurity correlations is dominated by a cross-term proportional to $J_{\mathrm{sd}} J_{0}$ [cf. fourth line in Eq. (12b)]. Thus, the sign of the frequency renormalization depends on the relative signs of the coupling constants $J_{\mathrm{sd}}$ and $J_{0}$. In principle, this allows a determination of the sign of the magnetic coupling constant $J_{\text {sd }}$ which cannot be obtained directly, e.g., by measuring the giant Zeeman splitting of excitons ${ }^{42}$.

\section{CONCLUSION}

We have investigated the influence of non-magnetic impurity scattering at $\mathrm{Mn}$ impurities on the spin dynamics in $\mathrm{Cd}_{1-x} \mathrm{Mn}_{x} \mathrm{Te}$ diluted magnetic semiconductors. To this end, we have developed a quantum kinetic theory taking the magnetic and non-magnetic carrier-impurity correlations into account. The Markov limit of the quantum kinetic equation is derived in order to distinguish the Markovian dynamics from genuine quantum kinetic effects.

In contrast to earlier studies ${ }^{25,27,37,40}$ in which only the magnetic contribution to the carrier-impurity interaction has been considered, some non-Markovian effects, such as a non-monotonic spin transfer between carriers and impurities, are strongly suppressed in the case of the conduction band of a $\mathrm{Cd}_{1-x} \mathrm{Mn}_{x}$ Te quantum well, while other features stemming from non-Markovian dynamics are enhanced, such as the large finite stationary value of the spin polarization in a magnetic field reached at long times. The reason for the suppression in the former case is that the non-magnetic impurity scattering leads to a strong redistribution of carriers in $\mathbf{k}$-space away from the states at $\mathbf{k}=0$. Since memory effects are particularly strong for carriers in proximity to the band edge ${ }^{27}$, this redistribution leads to spin dynamics that are well described by Markovian rate equations. The redistribution of the carriers implies an increase of their kinetic energies which is provided by a build-up of (negative) carrier-impurity correlation energy and which cannot be described by a mean-field or semiclassical approximation. We also provide analytic expressions for the correlation energies in the form of functionals of the spin-up and spin-down carrier occupations. Numerical calculations confirm that these expressions indeed describe the correlation energies obtained from the full quantum kinetic theory very well.

Even though doping with magnetic impurities unavoidably also provides a contribution to non-magnetic impurity scattering, there can still be situations where the latter is too weak to influence the spin dynamics and to suppress otherwise visible non-Markovian effects. This is substantiated by a model study of a $\mathrm{Cd}_{1-x} \mathrm{Mn}_{x}$ Te quantum well with degenerate valence bands, where the spin polarization exhibits a non-monotonic time dependence in the form of oscillations, while the Markovian treatment predicts a simple exponential decay. Further investigations using a more realistic valence band structure are needed in order to make more precise predictions about possible non-Markovian features in the hole spin dynamics in DMS.

In the presence of an external magnetic field parallel to the initial carrier spin (Faraday geometry), earlier studies $^{40}$ that did not consider non-magnetic impurity scattering predicted that the asymptotic value of the car- 
rier spins in the conduction band of a DMS quantum well at long times $t$ are significantly different in quantum kinetic and Markovian calculations. This was attributed to a broadening of the distribution of the scattered electrons due to the build-up of strong carrier-impurity correlations, which, because of the correlation energy, leads to a non-conservation of single-particle energies. The broadening results in an occupation of states by electrons whose back-scattering to the original band is strongly suppressed due to the lack of states with matching singleparticle energies. This induces a bias between spin-flip scattering from the spin-up to the spin-down subband and vice versa. In the presence of a strong non-magnetic carrer-impurity interaction, the correlation energy becomes much larger and with it also the broadening of the scattered carrier distribution and the deviations of the asymptotic value of the carrier spin polarization from its value obtained in Markovian calculations.

In the Voigt geometry, where the initial carrier spin polarization is perpendicular to the external field, the carrier spin precesses about the effective magnetic field comprised of the external field and the mean field due to the impurity magnetization. There, the carrier-impurity correlations lead to a renormalization of the spin precession frequencies. An analytic expression for this renormalization is presented and it is found to be of a similar form as the expression for the correlation energies. The nonmagnetic carrier-impurity interaction influences the frequency renormalization via a cross-term which vanishes if either the magnetic or the non-magnetic carrier-impurity interaction is neglected. In the case of the conduction band of $\mathrm{Cd}_{1-x} \mathrm{Mn}_{x} \mathrm{Te}$, the magnetic and non-magnetic contributions to the frequency renormalization have opposite signs. A measurement of the frequency renormalization can therefore indicate the sign of the exchange interaction. For magnetic fields at which the renormalization due to the magnetic correlations is particularly strong, the magnetic and non-magnetic contributions almost cancel each other. However, in most situations, the purely magnetic contribution is relatively weak ${ }^{39}$, so that the cross-term dominates the total frequency renormalization. The order of magnitude of the frequency renormalization for the cases considered here is about a few percent of the mean-field precession frequency.

To summarize, the influence of the non-magnetic impurity scattering on the spin dynamics in DMS is two-fold: First, it leads to a significant redistribution of carriers in k-space, which facilitates the suppression of some nonMarkovian effects in certain situations. Second, it causes a formation of strong many-body correlations between carriers and impurities, which result in large correlation energies and a significant renormalization of the carrier spin precession frequency.

\section{ACKNOWLEDGMENTS}

We gratefully acknowledge the financial support from the Universidad de Buenos Aires, project UBACyT 20142017 No. 20020130100514BA, and from CONICET, project PIP 11220110100091.

\section{Appendix A: Reduced set of equations of motions}

The equations of motions for the variables defined in Eq. (6) are:

$$
\begin{aligned}
\frac{\partial}{\partial t}\left\langle S^{l}\right\rangle & =\sum_{i m} \epsilon_{l i m} \omega_{\mathrm{Mn}}^{i}\left\langle S^{m}\right\rangle+\frac{J_{\mathrm{sd}}}{V} \sum_{\mathbf{k} \mathbf{k}^{\prime}} \sum_{i m} \epsilon_{l i m} \operatorname{Re}\left\{Q_{m \mathbf{k}}^{i \mathbf{k}^{\prime}}\right\} \\
\frac{\partial}{\partial t} n_{\mathbf{k}_{1}} & =\frac{J_{\mathrm{sd}} N_{\mathrm{Mn}}}{\hbar V^{2}} \sum_{\mathbf{k}} \sum_{i} 2 \operatorname{Im}\left\{Q_{i \mathbf{k}_{1}}^{i \mathbf{k}}\right\}+\frac{J_{0} N_{\mathrm{imp}}}{\hbar V^{2}} \sum_{\mathbf{k}} 2 \operatorname{Im}\left\{\bar{C}_{\mathbf{k}_{1}}^{0 \mathbf{k}}\right\}, \\
\frac{\partial}{\partial t} s_{k_{1}}^{l} & =\sum_{i j} \epsilon_{l i j} \omega_{\mathrm{e}}^{i} s_{\mathbf{k}_{1}}^{j}+\frac{J_{\mathrm{sd}} N_{\mathrm{Mn}}}{\hbar V^{2}} \sum_{\mathbf{k}}\left[\frac{1}{2} \operatorname{Im}\left\{Q_{l \mathbf{k}_{1}}^{0 \mathbf{k}}\right\}+\sum_{i j} \epsilon_{l i j} \operatorname{Re}\left\{Q_{i \mathbf{k}_{1}}^{j \mathbf{k}}\right\}\right]+\frac{J_{0} N_{\mathrm{imp}}}{\hbar V^{2}} \sum_{\mathbf{k}} 2 \operatorname{Im}\left\{\bar{C}_{\mathbf{k}_{1}}^{l \mathbf{k}}\right\} \\
\frac{\partial}{\partial t} Q_{l \mathbf{k}_{1}}^{0 \mathbf{k}_{2}} & =-i\left(\omega_{\mathbf{k}_{2}}-\omega_{\mathbf{k}_{1}}\right) Q_{l \mathbf{k}_{1}}^{0 \mathbf{k}_{2}}+\sum_{i i^{\prime}} \epsilon_{l i i^{\prime}} \omega_{\mathrm{Mn}}^{i} Q_{i^{\prime} \mathbf{k}_{1}}^{0 \mathbf{k}_{2}}+\frac{i}{\hbar} b_{l \mathbf{k}_{1}}^{0 \mathbf{k}_{2} I}+\frac{i}{\hbar} b_{l \mathbf{k}_{1}}^{0 \mathbf{k}_{2}}{ }^{i m p}, \\
\frac{\partial}{\partial t} Q_{l \mathbf{k}_{1}}^{j \mathbf{k}_{2}} & =-i\left(\omega_{\mathbf{k}_{2}}-\omega_{\mathbf{k}_{1}}\right) Q_{l \mathbf{k}_{1}}^{j \mathbf{k}_{2}}+\sum_{i i^{\prime}} \epsilon_{j i i^{\prime}} \omega_{\mathrm{e}}^{i} Q_{l \mathbf{k}_{1}}^{i^{\prime} \mathbf{k}_{2}}+\sum_{i i^{\prime}} \epsilon_{l i i^{\prime}} \omega_{\mathrm{Mn}}^{i} Q_{i^{\prime} \mathbf{k}_{1}}^{j \mathbf{k}_{2}}+\frac{i}{\hbar} b_{l \mathbf{k}_{1}}^{j \mathbf{k}_{2} I}+\frac{i}{\hbar} b_{l \mathbf{k}_{1}}^{j \mathbf{k}_{2}}{ }^{i m p} \\
\frac{\partial}{\partial t} \bar{C}^{0 \mathbf{k}_{1}} & =-i\left(\omega_{\mathbf{k}_{2}}-\omega_{\mathbf{k}_{1}}\right) \bar{C}_{\mathbf{k}_{1}}^{0 \mathbf{k}_{2}}+\frac{i}{\hbar} c_{\mathbf{k}_{1}}^{0 \mathbf{k}_{2} I}+\frac{i}{\hbar} c_{\mathbf{k}_{1}}^{0 \mathbf{k}_{2} \mathrm{sd}}, \\
\frac{\partial}{\partial t} \bar{C}^{j \mathbf{k}_{1}} & =-i\left(\omega_{\mathbf{k}_{2}}-\omega_{\mathbf{k}_{1}}\right) \bar{C}_{\mathbf{k}_{1}}^{j \mathbf{k}_{2}}+\sum_{i i^{\prime}} \epsilon_{j i i^{\prime}} \omega_{\mathrm{e}}^{i} C_{\mathbf{k}_{1}}^{i^{\prime} \mathbf{k}_{2}}+\frac{i}{\hbar} c_{\mathbf{k}_{1}}^{j \mathbf{k}_{2} I}+\frac{i}{\hbar} c_{\mathbf{k}_{1}}^{j \mathbf{k}_{2} \mathrm{sd}}
\end{aligned}
$$


with

$$
\begin{aligned}
& b_{l \mathbf{k}_{1}}^{0 \mathbf{k}_{2} I}=J_{\mathrm{sd}} \sum_{i}\left[\operatorname{Re}\left\{\left\langle S^{i} S^{l}\right\rangle\right\}\left(s_{\mathbf{k}_{2}}^{i}-s_{\mathbf{k}_{1}}^{i}\right)+i \sum_{m} \epsilon_{i l m} \frac{\left\langle S^{m}\right\rangle}{2}\left(\left(1-n_{\mathbf{k}_{1}}\right) s_{\mathbf{k}_{2}}^{i}+\left(1-n_{\mathbf{k}_{2}}\right) s_{\mathbf{k}_{1}}^{i}\right)+\left\langle S^{i}\right\rangle\left(s_{\mathbf{k}_{1}}^{l} s_{\mathbf{k}_{2}}^{i}-s_{\mathbf{k}_{1}}^{i} s_{\mathbf{k}_{2}}^{l}\right)\right], \\
& b_{l \mathbf{k}_{1}}^{j \mathbf{k}_{2} I}=J_{\mathrm{sd}} \sum_{i}\left[\operatorname{Re}\left\{\left\langle S^{i} S^{l}\right\rangle\right\}\left[\delta_{i j}\left(\frac{n_{\mathbf{k}_{2}}}{4}-\frac{n_{\mathbf{k}_{1}}}{4}\right)+\frac{i}{2} \epsilon_{i j k}\left(s_{\mathbf{k}_{1}}^{k}+s_{\mathbf{k}_{2}}^{k}\right)\right]+\frac{i}{2} \sum_{m} \epsilon_{i l m}\left\langle S^{m}\right\rangle\left[\delta_{i j} \frac{n_{\mathbf{k}_{1}}+n_{\mathbf{k}_{2}}-n_{\mathbf{k}_{1}} n_{\mathbf{k}_{2}}}{4}\right.\right. \\
& \left.\left.+\delta_{i j} \mathbf{s}_{\mathbf{k}_{1}} \cdot \mathbf{s}_{\mathbf{k}_{2}}-\left(s_{\mathbf{k}_{1}}^{i} s_{\mathbf{k}_{2}}^{j}+s_{\mathbf{k}_{2}}^{i} s_{\mathbf{k}_{1}}^{j}\right)+\frac{i}{2} \epsilon_{i j k}\left(\left(1-n_{\mathbf{k}_{1}}\right) s_{\mathbf{k}_{2}}^{k}-\left(1-n_{\mathbf{k}_{2}}\right) s_{\mathbf{k}_{1}}^{k}\right)\right]\right], \\
& b_{l \mathbf{k}_{1}}^{0 \mathbf{k}_{2}}{ }^{\text {imp }}=J_{0}\left\langle S^{l}\right\rangle\left(n_{\mathbf{k}_{2}}-n_{\mathbf{k}_{1}}\right) \text {, } \\
& b_{l \mathbf{k}_{1}}^{j \mathbf{k}_{2}} \text { imp }=J_{0}\left\langle S^{l}\right\rangle\left(s_{\mathbf{k}_{2}}^{j}-s_{\mathbf{k}_{1}}^{j}\right), \\
& c_{\mathbf{k}_{1}}^{0 \mathbf{k}_{2} I}=J_{0}\left(n_{\mathbf{k}_{2}}-n_{\mathbf{k}_{1}}\right), \\
& c_{\mathbf{k}_{1}}^{j \mathbf{k}_{2} I}=J_{0}\left(s_{\mathbf{k}_{2}}^{j}-s_{\mathbf{k}_{1}}^{j}\right), \\
& c_{\mathbf{k}_{1}}^{0 \mathbf{k}_{2} \mathrm{sd}}=J_{\mathrm{sd}}\left\langle S^{i}\right\rangle\left(s_{\mathbf{k}_{2}}^{i}-s_{\mathbf{k}_{1}}^{i}\right), \\
& c_{\mathbf{k}_{1}}^{j \mathbf{k}_{2}}{ }^{\mathrm{sd}}=J_{\mathrm{sd}}\left[\frac{1}{4}\left\langle S^{j}\right\rangle\left(n_{\mathbf{k}_{2}}-n_{\mathbf{k}_{1}}\right)+\frac{i}{2} \epsilon_{i j k}\left\langle S^{i}\right\rangle\left(s_{\mathbf{k}_{2}}^{k}+s_{\mathbf{k}_{1}}^{k}\right)\right] .
\end{aligned}
$$

1 M. N. Baibich, J. M. Broto, A. Fert, F. N. Van Dau, F. Petroff, P. Etienne, G. Creuzet, A. Friederich, and J. Chazelas, Phys. Rev. Lett. 61, 2472 (1988).

2 S. A. Wolf, D. D. Awschalom, R. A. Buhrman, J. M. Daughton, S. von Molnár, M. L. Roukes, A. Y. Chtchelkanova, and D. M. Treger, Science 294, 1488 (2001).

3 S. Datta and B. Das, Appl. Phys. Lett. 56, 665 (1990).

4 D. Awschalom and M. Flatté, Nat. Phys. 3, 153 (2007).

5 I. Žutić, J. Fabian, and S. Das Sarma, Rev. Mod. Phys. 76, 323 (2004).

6 T. Dietl, Nat. Mater. 9, 965 (2010).

7 H. Ohno, Nat. Mater. 9, 952 (2010).

8 T. Dietl and H. Ohno, Rev. Mod. Phys. 86, 187 (2014).

9 T. Dietl, H. Ohno, F. Matsukura, J. Cibert, and D. Ferrand, Science 287, 1019 (2000).

10 Z. Ben Cheikh, S. Cronenberger, M. Vladimirova, D. Scalbert, F. Perez, and T. Wojtowicz, Phys. Rev. B 88, 201306 (2013).

11 Y. S. Chen, M. Wiater, G. Karczewski, T. Wojtowicz, and G. Bacher, Phys. Rev. B 87, 155301 (2013).

12 S. Cronenberger, M. Vladimirova, S. V. Andreev, M. B. Lifshits, and D. Scalbert, Phys. Rev. Lett. 110, 077403 (2013).

13 J. Debus, V. Y. Ivanov, S. M. Ryabchenko, D. R. Yakovlev, A. A. Maksimov, Y. G. Semenov, D. Braukmann, J. Rautert, U. Löw, M. Godlewski, A. Waag, and M. Bayer, Phys. Rev. B 93, 195307 (2016).

14 J. Debus, A. A. Maksimov, D. Dunker, D. R. Yakovlev, E. V. Filatov, I. I. Tartakovskii, V. Y. Ivanov, A. Waag, and M. Bayer, phys. stat. sol. (b) 251, 1694 (2014).

15 A. A. Maksimov, D. R. Yakovlev, J. Debus, I. I. Tartakovskii, A. Waag, G. Karczewski, T. Wojtowicz, J. Kossut, and M. Bayer, Phys. Rev. B 82, 035211 (2010).
16 F. Perez, J. Cibert, M. Vladimirova, and D. Scalbert, Phys. Rev. B 83, 075311 (2011).

17 M. D. Kapetanakis, I. E. Perakis, K. J. Wickey, C. Piermarocchi, and J. Wang, Phys. Rev. Lett. 103, 047404 (2009).

18 M. D. Kapetanakis and I. E. Perakis, Phys. Rev. Lett. 101, 097201 (2008).

19 H. Krenn, K. Kaltenegger, T. Dietl, J. Spałek, and G. Bauer, Phys. Rev. B 39, 10918 (1989).

20 S. A. Crooker, D. D. Awschalom, J. J. Baumberg, F. Flack, and N. Samarth, Phys. Rev. B 56, 7574 (1997).

21 P. Barate, S. Cronenberger, M. Vladimirova, D. Scalbert, F. Perez, J. Gòmez, B. Jusserand, H. Boukari, D. Ferrand, H. Mariette, J. Cibert, and M. Nawrocki, Phys. Rev. B 82, 075306 (2010).

22 J. H. Jiang, Y. Zhou, T. Korn, C. Schüller, and M. W. Wu, Phys. Rev. B 79, 155201 (2009).

23 H. Ohno, A. Shen, F. Matsukura, A. Oiwa, A. Endo, S. Katsumoto, and Y. Iye, Appl. Phys. Lett. 69, 363 (1996).

24 R. Fiederling, M. Keim, G. Reuscher, W. Ossau, G. Schmidt, A. Waag, and L. W. Molenkamp, Nature 402, 787 (1999).

25 C. Thurn, M. Cygorek, V. M. Axt, and T. Kuhn, Phys. Rev. B 87, 205301 (2013).

26 M. Cygorek and V. M. Axt, Phys. Rev. B 90, 035206 (2014).

27 M. Cygorek and V. M. Axt, Semicond. Sci. Technol. 30, 085011 (2015).

28 M. Cygorek, P. I. Tamborenea, and V. M. Axt, Phys. Rev. B 93, 205201 (2016).

29 J. Kondo, Prog. Theor. Phys. 32, 37 (1964).

30 J. Kossut, phys. stat. sol. (b) 72, 359 (1975). 
31 A. Twardowski, M. Nawrocki, and J. Ginter, phys. stat. sol. (b) 96, 497 (1979).

32 R. Bouzerar and G. Bouzerar, EPL (Europhysics Letters) 92, 47006 (2010).

33 M. W. Wu, J. H. Jiang, and M. Q. Weng, Phys. Rep. 493, 61 (2010).

34 R. J. Elliott, Phys. Rev. 96, 266 (1954).

35 M. I. D'yakonov and V. I. Perel', Zh. Eksp. Teor. Fiz. 60, 1954 (1971), [Sov. Phys. JETP 33, 1053 (1971)].

36 C. Thurn and V. M. Axt, Phys. Rev. B 85, 165203 (2012).

37 C. Thurn, M. Cygorek, V. M. Axt, and T. Kuhn, Phys. Rev. B 88, 161302(R) (2013).

38 M. Cygorek and V. M. Axt, Journal of Physics: Conference Series 647, 012042 (2015).

39 M. Cygorek, P. I. Tamborenea, and V. M. Axt, Phys. Rev. B 93, 035206 (2016); 94, 079906 (2016).
40 M. Cygorek, F. Ungar, P. I. Tamborenea, and V. M. Axt, submitted to Proceedings of SPIE, Optics + Photonics: Nanoscience + Engineering, Spintronics IX (2016).

41 F. Ungar, M. Cygorek, P. I. Tamborenea, and V. M. Axt, Phys. Rev. B 91, 195201 (2015).

42 J. Kossut and J. Gaj, eds., Introduction to the Physics of Diluted Magnetic Semiconductors, Springer Series in Materials Science No. 144 (Springer, Berlin, 2011).

43 F. Long, W. E. Hagston, P. Harrison, and T. Stirner, Journal of Applied Physics 82, 3414 (1997).

44 S.-K. Chang, A. V. Nurmikko, J.-W. Wu, L. A. Kolodziejski, and R. L. Gunshor, Phys. Rev. B 37, 1191 (1988).

45 J. Schilp, T. Kuhn, and G. Mahler, Phys. Rev. B 50, 5435 (1994).

46 C. Fürst, A. Leitenstorfer, A. Laubereau, and R. Zimmermann, Phys. Rev. Lett. 78, 3733 (1997). 